\title{
Analysis of the expression and clinical significance of miR-382-5P in ovarian cancer based on biological information
}

peiyang wu ( $\nabla 31044492 @ q q . c o m$ )

affifiliated tumor hospital of guangxi medical university https://orcid.org/0000-0001-7358-1393

Li Li

guangxi medical university cancer hospital

\section{Research}

Keywords: Ovarian cancer, miR-382-5P, Targeted gene, Signal pathway, Bioinformatics

Posted Date: March 11th, 2020

DOI: https://doi.org/10.21203/rs.3.rs-16753/v1

License: 우 (i) This work is licensed under a Creative Commons Attribution 4.0 International License. Read Full License 


\section{Abstract}

Objective : To investigate the expression, potential function and clinical significance of miR-382-5P in ovarian cancer tissues .

Results: The expression of miR-382-5P is tissue-specific. Four target gene prediction softwares including miRPathDB ,DIANA ,miRWalk and starBase

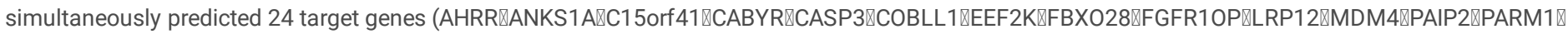

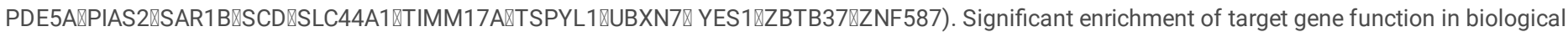
processes, cell composition and molecular functions, signal transduction pathways are significantly enriched in microRNAs in cancer, MAPK signaling pathway, Hepatitis B, FoxO signaling pathway, Non-small cell lung cancer, apoptosis, Colorectal cancer, Oxytocin signaling pathway. TCGA database data analysis did not indicate that there was a correlation between the expression of miR-382-5P and the clinicopathological parameters of ovarian cancer, but there was no significant difference $(P>0.05)$. The results of QRT-PCR in 72 ovarian cancer clinical specimens showed that the expression of miR-382-5P was

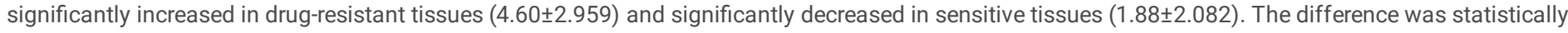
significant $(\mathrm{P}<0.05)$. Analyzing the follow-up data of 72 patients with ovarian cancer in our hospital, we found that the expression level of miR-382-5P in ovarian cancer patients was correlated with the degree of drug resistance, and the difference was statistically significant $(P<0.05)$. The expression level of miR-382-5P, drug resistance, recurrence and treatment response in patients with ovarian cancer were correlated with their overall survival (OS), and the difference was statistically significant $(P<0.05)$. The expression level of miR-382-5P, drug resistance, treatment response, recurrence and residual lesion size in patients with ovarian cancer were correlated with their progression-free survival (PFS), the difference was statistically significant ( $<<0.05)$. Multidrug resistance is an independent risk factor affecting the prognosis of ovarian cancer, the difference was statistically significant $(P<0.05)$

Conclusion $₫$ After comprehensive analysis, it is found that miR-382-5P may become a new target for the treatment of multidrug resistant ovarian cancer. The signal pathways of MAPK, Fox-1,apoptosis and so on may be the main mechanism of miR-382-5P mediating the occurrence and development of ovarian cancer, and lay a certain foundation for the research of targeted miR-382-5P in the treatment of multidrug resistant ovarian cancer.

\section{Introduction}

Ovarian cancer is one of the most common malignant tumors in gynecology. Ovarian cancer has become a hidden killer because of its deep anatomical location, which is often advanced at the time of consultation. Its morbidity and mortality are the second and the first in gynecological malignant tumors ${ }^{\text {[1] }}$ The main treatment is tumor reduction combined with platinum-based post-operative chemotherapy. The emergence of PARP inhibitors significantly prolongs the survival time of ovarian cancer patients, but drug resistance remains one of the main causes of cancer recurrence.microRNAs induce drug resistance by regulating gene stability ${ }^{[2]}$. A series of nucleases obtained mature miR-382-5P and miR-382-3P by cutting and editing miR-382, in which miR-382-5P was located in the long arm of chromosome 14. In recent years, many studies have shown that the expression of miR-382-5P is abnormal in oral squamous cell carcinoma ${ }^{[3]}$,breast cancer ${ }^{[4]}$,glioma ${ }^{[5,6]}$,acute promyelocytic leukemia ${ }^{[7,8]}$, primary myelofibrosis ${ }^{[9]}$,epidural fibrosis ${ }^{[10]}$,primary liver cancer ${ }^{[11]}$, atherosclerosis ${ }^{[12]}$, cervical cancer ${ }^{[13]}$, miR-382 is abnormal in non-small cell lung cancer ${ }^{[14,15]}$, osteosarcoma ${ }^{[16]}$, diabetic nephropathy ${ }^{[17]}$, IgA nephropathy ${ }^{[18]}$ prostate cancer ${ }^{[19]}$, ovarian cacner ${ }^{[20]}$, colorectal cancer ${ }^{[21,22]}$, infantile hemangioma ${ }^{[23]}$,primary liver cancer ${ }^{[24]}$,schizophrenia ${ }^{[25]}$, which are closely related to the proliferation, differentiation, migration, invasion, drug resistance and other biological processes of tumor cells. However, the expression and clinical significance of miR-382-5P in ovarian cancer have not been reported. Previous studies in vitro showed that the expression of miR-382-5P was upregulated in ovarian cancer drug-resistant cell line SKOV3DDP than in parent cell line SKOV3, and confirmed that HIPK3 was the target gene of miR-382-5P [26]. Therefore, this study intends to predict the target gene of miR-382-5P through biological analysis, enrich its target gene and download HIPK3 related genes, perform GO functional annotation analysis and KEGG signal transduction pathway enrichment analysis, and analyze the correlation between miR-382-5P and clinicopathological parameters and prognosis of ovarian cancer by combining the expression of miR-382-5P in clinical tissue samples and follow-up data,providing a theoretical basis for the follow-up study of the function and mechanism of miR-382-5P, which is expected to become one of the new targets for the treatment of drug-resistant ovarian cancer.

\section{Materials And Methods}

1.1 Bioinformatics analysis of hsa-miR-382-5p

\subsubsection{Getting basic information of hsa-miR-382-5p}

Three online databases,namely NCBI(https://www.ncbi.nlm.nih.gov/), miRbase(http://www.mirbase.org/),UCSC(http://genome.ucsc.edu/),were used to search for basic information such as sequence number, chromosome location, length and base sequence of hsa-miR-382-5p.

1.1.2 Using pubMed to search the related literature of hsa-miR-382-5p

In PubMed database (http://www.ncbi.nlm.nih.gov/pubmed/), " hsa-miR-382-5p " is used as the key word to search, and the relevant literature of hsa-miR-382$5 p$ is carefully read.

1.1.3 Predicting target genes of hsa-miR-382-5p

miRPathDB(https://mpd.bioinf.uni-sb.de/),

DIANA(http://diana.imis.athena-innovation.gr),

miRWalk(http://mirwalk.umm.uni-heidelberg.de), 
starBase(http://starbase.sysu.edu.cn/), online target gene prediction software, was used to predict target gene. The target genes predicted by each software were obtained by different calculation methods through searching for "hsa-mir-382-5p" as the search term.

VENNY 2.1 (http://bioinfogp.cnb.csic.es/tools/venny/index.html) online software takes at least three target genes predicted by prediction software at the same time, and carries on the intersection, in order to reduce its false positive rate

1.1.4 cBioPortal database

Using cBioPortal database (https://www.cbioportal.org/) to search for HIPK3-related genes, the criteria for screening were: Q value < 0.001 .

1.1.5 KEGG analysis and GO analysis

A online software, DAVID (https://david.ncifcrf.gov/) was using. signal transduction pathway enrichment analysis (KEGG analysis) and function annotation analysis (GO analysis) were carried out for the target genes of miR-382-5p obtained from the intersection and HIPK3 related genes. The criteria for entry selection were: $P$ value $<0.05$

1.1.6 Data analysis of TCGA database

The official download tool GDCapps provided by TCGA database (https://portal.gdc.cancer.gov/) was used to download the related data and clinical data of ovarian cancer microRNA-Seq. The related data of microRNA-Seq were processed by R software, and the clinical pathological parameters were analyzed with clinical data.

1.2 Detection of hsa-miR-382-5p expression in clinical specimens of ovarian cancer and analysis of clinical data

1.2.1 Detection of hsa-miR-382-5p expression in clinical specimens of ovarian cancer by QRT-PCR

Cancer tissue specimens from patients with epithelial ovarian cancer treated surgically in Tumor Hospital Affiliated to Guangxi Medical University from August 22, 2001 to December 24, 2012 were collected. Among them, 30 patients were resistant to chemotherapy and 42 patients were sensitive to chemotherapy. All patients with ovarian cancer were diagnosed as epithelial ovarian cancer by intraoperative frozen and postoperative pathology, including serous ovarian cancer and mucinous ovarian cancer predominantly. More than $80 \%$ of the tumors are in tumors. FIGO stage is Ic-IV. Patients with ovarian cancer included in the study have undergone at least three-cycle first-line chemotherapy. Inclusion criteria of chemotherapeutic resistance group of epithelial ovarian cancer: The time from the end of 6 courses of ideal subtraction surgery and combination of paclitaxel and carboplatin chemotherapy to the recurrence of ovarian cancer was less than or equal to 6 months. Inclusion criteria of chemosensitivity group for epithelial ovarian cancer: The time from the end of 6 courses of ideal subtraction surgery and combined paclitaxel and carboplatin chemotherapy to the recurrence of ovarian cancer was longer than 12 months, or no imaging findings suggested that the recurrence of ovarian cancer. The basis of recurrence of ovarian cancer: color Doppler, CT, MRI, PET/CT and other imaging indicators of recurrence of ovarian cancer. All the candidates signed the informed consent and were examined by the ethics committee of Guangxi Medical UniversitymiRNeasy Mini Kit】miScript II Reverse Transcription Kit \miScript SYBR Green PCR Kit were purchased from Qiagen, Germany. The upstream primers of PCR were synthesized by Yingweijie (Guangzhou) Trading Co., Ltd. The downstream primers used the universal reverse primers provided by

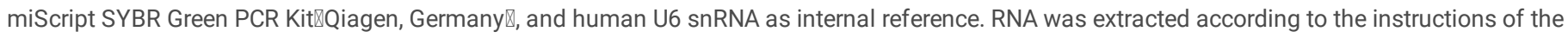

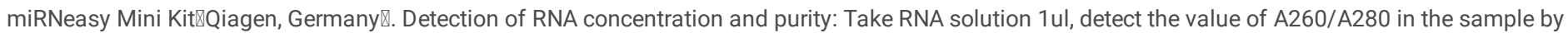
enzyme labeling instrument, calculate the concentration. Samples with satisfactory concentration and purity were retrieved and amplified by miScript II

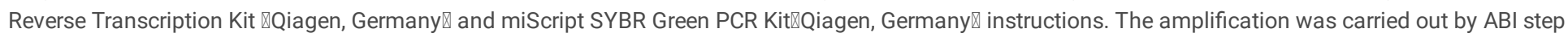
one plus PCR. The 20ul reaction system contained 10ul 2 * QuanteTect SYBR Green PCR Master Mix, 2ul 10 * miScript Universal Primer, 2ul 10 * miScript Primer Assay, 1 ul template cDNA, 5ul RNase-free Water. For U6 internal reference gene, relative quantitative method was used to detect the expression of target gene, and $2^{-\triangle \triangle C t}$ was used to evaluate the expression level of miR-382-5p. Primer sequence: miR-382-5p upstream sequence 5'-GAAGTTGTTCGTGGATTC-3', U6 upstream sequence 5'-CAAGGATGACACGCAAATTCG-3'.

1.2.2 Statistical methods for analysis of the correlation between miR-382-5p expression level and clinicopathological parameters and prognosis in ovarian cancer

All statistical analysis were processed by SPSS 23.0. To analyze the correlation between miR-382-5p expression level and clinicopathological parameters of ovarian cancer, chi-square test was used, survival curve was Kaplan-Meier, Log-rank test was used to compare, and Cox proportional risk model was used to analyze single factor and multiple factors to determine the risk factors affecting prognosis. Test level alpha $=0.05$ (double tail). Diagnostic tests were performed with diagnostic indices and ROC curves. R 3.6.1 draws forest maps of regression analysis results.

\section{Results}

2.1 Biological analysis of miR-382-5P

2.1.1 Basic information of miR-382-5P

Three online databases, including NCBI, miRBase and UCSC, were searched. It was found that the sequence number of miR-382-5P was MIMAT0000737, located in a cluster of chromosome 14q32.31. The mature gene locus were chr14:101,054,316-101,054,337, and the base sequence was GAAGUUGUUCGUGGUGGAUUCG , with a length of $22 \mathrm{nt}$.(Fig.1)

Page $3 / 31$ 
2.1.2 hsa-miR-382-5P participates in disease regulation

Using PubMed to retrieve the related literature of miR-382-5P, we found that miR-382-5P was up-regulated in acute promyelocytic leukemia

${ }^{[7,8]}$, atherosclerosis ${ }^{[12]}$,breast cancer ${ }^{[4]}$,epidural fibrosis ${ }^{[10]}$, primary myelofibrosis ${ }^{[9]}$, primary liver cancer ${ }^{[11]}$, cervical cancer ${ }^{[13]}$, but down-regulated in glioma $[5,6]$,oral squamous cell carcinoma ${ }^{[3]}{ }^{\text {, miR-38 }} 38$ was down-regulated in non-small cell lung cancer ${ }^{[14,15]}$, osteosarcoma ${ }^{[16]}$, prostate cancer ${ }^{[19]}$, ovarian cancer ${ }^{[20]}$, primary liver cancer ${ }^{[24]}$, colorectal cancer ${ }^{[21,22]}$, while schizophrenia ${ }^{[25]}$, diabetic nephropathy ${ }^{[17]}$, IgA nephropathy ${ }^{[18]}$, infantile hemangioma ${ }^{[23]}$. Thus, the expression of miR-382-5P is tissue-specific, and it is involved in the regulation of cell viability, migration, invasion, angiogenesis, proliferation, cell differentiation, oxidative stress and other biological behaviors. (Table 1)

2.1.3 Prediction of target genes of hsa-miR-382-5p

Four on-line predictive softwares for target genes of microRNAs, such as miRPathDB, DIANA, miRWalk and starBase, were used to predict 6599 , 746, 1462 and 1625 target genes respectively. 24 target genes

(AHRR,ANKS1A,C15orf41,CABYR,CASP3,COBLL1,EEF2K,FBX028,FGFR1OP,LRP12,MDM4,PAIP2,PARM1,PDE5A,PIAS2,SAR1B,SCD,SLC44A1,TIMM17A,TSPYL1, were obtained from the above four predictive softwares, which accounted for $0.3 \%$ of the prediction software respectively, and $67,28,216$ and 116 target genes were obtained from the intersection of three prediction software, which accounted for $0.9 \%, 0.8 \%, 2.9 \%$ and $1.5 \%$ of the target gene prediction software respectively. (Fig. 2-6)

2.1.4 Analysis of GO function annotation of miR-382-5p target gene

GO function annotation analysis of 116 target genes predicted by three predictive software, including miRWalk, starBase and miRPathDB, revealed that their target genes were involved in biological processes, such as peptidyl-serine phosphorylation, positive regulation of execution phase of apoptosis, positive regulation of lipopolysaccharide-mediated signaling pathway, negative regulation of apoptotic process. Molecular functions such as protein serine/threonine kinase activity, ATP binding, siRNA binding. Cell composition such as cytoplasm, cell membrane, cell nucleus. $(P<0.05)(T a b l e ~ 2-4)$

\subsection{5 cBioPort database}

Using cBioPort database to query and download HIPK3 related genes, the screening criteria were Q value $<0.001$, and 822 related genes such as QSER1, CAPRIN1, EHF, PIK3CB, ZBTB38, NIPAL2,etc were obtained.(As shown in the supplementary)

\subsubsection{KEGG analysis of HIPK3-related genes}

KEGG signal transduction pathway was enriched for HIPK3 related genes. It was found that HIPK3 related genes were significantly enriched in Signaling pathways regulating pluripotency of stem cells, Platelet activation and Ras signaling pathway. $(\mathrm{P}<0.05)($ Table 5$)$

\subsubsection{KEGG analysis of miR-382-5p target gene}

KEGG signal transduction pathway was enriched in 116 target genes predicted by three predictive software, including miRWalk, starBase and miRPathDB. It was found that miR-382-5p target genes were significantly enriched in microRNAs in cancer, MAPK signaling pathway, Hepatitis B, FoxO signaling pathway, Non-small cell lung cancer, apoptosis, Colorectal cancer, Oxytocin signaling pathway. $(P<0.05)($ Table 6$)$

\subsubsection{Data analysis results of TCGA database}

The TCGA database downloaded the related data of microRNA-Seq and clinical data of ovarian cancer. Among them, 489 cases which detected the expression of miR-382-5p were divided into 244 Cases of miR-382-5p low-expression group and 245 cases of miR-382-5p high-expression group according to the median. Statistical analysis showed that there was no correlation between the expression of miR-382-5p and age, unilateral or bilateral, histological grade, FIGO stage, lymph node metastasis, vascular invasion, drug resistance, recurrence, survival, treatment response, residual tumor size, and tumor diameter, there was no significant difference $(P>0.05)$. (Table 7)

2.2 Detection of miR-382-5p expression in clinical specimens of ovarian cancer and analysis of clinical data

2.2.1 Detection of miR-382-5p expression in clinical specimens of ovarian cancer by QRT-PCR

QRT-PCR results showed that miR-382-5p was significantly up-regulated in resistant ovarian cancer tissues (4.60 \pm 2.959$)$, down-regulated in sensitive ovarian cancer tissues (1.88 \pm 2.082$)$, and 95\% confidence interval (1.456,3.983). The difference was statistically significant $(P<0.05)$. (Fig. 7)

2.2.2 Statistical analysis of the expression of miR-382-5p and its clinicopathological parameters and prognosis in ovarian cancer

The correlation between the expression level of miR-382-5p and clinicopathological parameters was analyzed. Statistical analysis showed that miR-382-5p was closely related to the drug resistance of ovarian cancer, there was significant difference $(P<0.05)$, but there was no correlation between miR-382-5p and age, histological grade, FIGO stage, pathological type, relapse, residual lesion size, survival status, treatment response of ovarian cancer patients, there was no significant difference $(P>0.05)$. (Table 8)

2.2.3 The correlation between the expression of miR-382-5P in ovarian cancer and its clinicopathological parameters and prognosis 
2.2.3.1 Kaplan-Meier method was used to analyze the the correlation between expression of miR-382-5P in ovarian cancer and its clinicopathological parameters and prognosis.

Kaplan-Meier method was used to analyze the correlation between the expression level of miR-382-5P in 72 ovarian cancer specimens and its clinicopathological parameters and prognosis. The results showed that OS in the low-expression group of miR-382-5P(83.313 \pm 13.743$)$ was significantly longer than that in the high-expression group of miR-382-5P (45.5 \pm 6.673$)$, the difference was statistically significant $(P<0.05)$; $P F S$ in the low expression group of miR-382-5P(68.014 \pm 13.30$)$ was significantly prolonged in the high expression group of miR-382-5P (26.932 \pm 5.914$)$, the difference was statistically significant $(P<0.05)$. OS in chemosensitivity group(71.494 \pm 9.549$)$ was significantly longer than that in chemoresistance group $(42.034 \pm 8.309)$, the difference was statistically significant $(P<0.05)$; PFS in chemosensitivity group $(60.784 \pm 9.126)$ was significantly longer than that in chemoresistance group(6.652 \pm 0.654$)$, the difference was statistically significant $(P<0.05)$. PFS in the group with residual lesion diameter less than $1 \mathrm{~cm}(47.199 \pm 8.245)$ was significantly longer than that in the group with diameter greater than $1 \mathrm{~cm}(17.724 \pm 3.518)$, the difference was statistically significant $(P<0.05)$, while there was no significant correlation between residual lesion diameter and OS $(P>0.05)$. PFS in non-recurrence group $\otimes 97 \pm 13.954 \rrbracket$ was significantly longer than that in recurrence group $(29.86 \pm 5.74)$, the difference was statistically significant $(P<0.05)$; OS in non-recurrence group $₫ 97 \pm 13.954 \llbracket$ was significantly longer than that

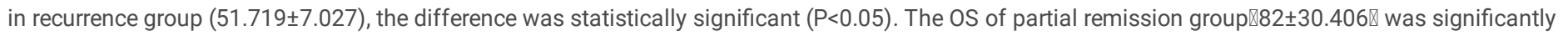
higher than that of complete remission group (71.299 \pm 10.014$)$, progression group (31.2 \pm 10.148$)$, stable condition group ( $30.6 \pm 5.240)$, the difference was statistically significant $(\mathrm{P}<0.05)$; PFS of complete remission group $(55.1 \pm 9.861)$ was significantly higher than that of partial remission group (35 \pm 0$)$, progression group (6.742 \pm 0.719$)$, and stable condition group (6.8 \pm 1.562$)$, the difference was statistically significant $(P<0.05)$. However, age, pathological type, histological grade, FIGO stage had no significant correlation with OS and PFS ( $>0.05)$. Therefore,it can be seen that low levels of miR-382-5P, chemosensitivity, residual lesions less than $1 \mathrm{~cm}$, no recurrence and complete remission in treatment response are positively correlated with PFS. Low levels of miR-382-5P, chemosensitivity, no recurrence and partial remission in treatment response are positively correlated with OS. (Fig. 8-25)

\subsubsection{Independent risk factors affecting the prognosis of ovarian cancer patients assessed by Cox proportional hazard model}

\subsection{Cox univariate and multivariate analysis was used to assess independent risk factors affecting the prognosis of patients with ovarian cancer}

Cox proportional hazard model was used to analyze the correlation between the expression level of miR-382-5P and clinicopathological parameters and the prognosis of 72 cases of ovarian cancer in our hospital. The independent risk factors affecting the prognosis of ovarian cancer patients were evaluated. Cox single factor analysis showed that stage II, III and IV were independent risk factors affecting the prognosis of ovarian cancer patients in FIGO staging, and stage IV was an independent risk factor affecting the prognosis of ovarian cancer patients. The risk of death was 5.953 times that of stage III, $95 \%$ confidence interval was $1.438-24.646$, stage III was 2.981 times that of stage II, $95 \%$ confidence interval was $0.909-9.78$, stage II was 3.151 times that of stage I, and $95 \%$ confidence interval was $0.702-14.15$. Drug resistance was an independent risk factor affecting the prognosis of ovarian cancer patients, its the risk of death in drug-resistant group was 2.075 times that of sensitive group , and 95\% confidence interval was 1.138-3.783. In the expression level of miR-382-5P in ovarian cancer, the high expression of miR-382-5P was an independent risk factor affecting the prognosis of ovarian cancer patients. The death risk of high expression group was 2.143 times that of low expression group, and the $95 \%$ confidence interval was $1.052-4.363$. In the treatment response of ovarian cancer, the stable disease group and disease progression group were influencing ovarian cancer. Independent risk factors for prognosis of patients in diseasestable group were 2.079 times higher than that in partial remission group, 95\% confidence interval was $0.774-5.583$, the risk of death in disease-progression group was 2.724 times higher than that in disease-stable group, and 95\% confidence interval was 1.23-6.032. In the recurrence of ovarian cancer, recurrence was an independent risk factor affecting the prognosis of patients with ovarian cancer, its the risk of death in recurrence group was 3.224 times that of no recurrence group , and the $95 \%$ confidence interval was $0.99-10.496$. Cox multivariate analysis showed that drug resistance was an independent risk factor affecting the prognosis of ovarian cancer patients. The risk of death in drug-resistant group was 2.149 times higher than that in sensitive group, and the $95 \%$ confidence interval was 1.092-4.23. Fig26. Table9.

\subsubsection{Diagnostic tests to determine the accuracy of miR-382-5P as an index of drug resistance in ovarian cancer}

In this study, we calculated the diagnostic indices and evaluated the accuracy of miR-382-5P as an index of multidrug resistance in ovarian cancer by using ROC curve. The results showed that sensitivity: $56 \%$, specificity: $91 \%$, positive predictive value: $93 \%$, negative predictive value: $48 \%$, positive likelihood ratio: 6.22, negative likelihood ratio: $48 \%$, Yoden index: $47 \%$, area under ROC curve: $70.5 \%$, P value: 0.003 . There was statistical significance. These results suggest that the use of miR-382-5P in the diagnosis of drug resistance in ovarian cancer has a good accuracy, and it can be used as an experimental diagnostic method for drug resistance in ovarian cancer. As shown in Figure. 27

\section{Discussion}

microRNAs were first found in Caenorhabditis elegans in the early $1990 s^{[27]}$.microRNAs is a single-stranded non-coding RNA with length of $19-25$ nt. By the principle of base complementary pairing, microRNAs can partially bind to the 3 'non-coding region of the mRNA to degrade or inhibit the translation process of the target gene, thereby negatively regulating the expression of the target gene ${ }^{[28]}$. Due to microRNAs are highly abundant, conservative and tissue-specific, they are involved in the regulation of gene regulation, cell development, various diseases and other biological processes ${ }^{[29]}$. Its participation in the regulation of biological processes is through direct targeting of target genes, which the binding of 5'end of miRNA to 3' UTR of target gene needs to consider many factors, such as sequence complementarity, sequence conservativeness, thermodynamic factors, site binding, UTR base distribution and so on. Therefore, in order to improve specificity and reduce false positive rate, we used four target gene prediction software, including miRPathDB, DIANA, miRWalk and starBase, to predict target genes by different calculation methods, and obtained 24 target genes(AHRR, ANKS1A, C15orf41, CABYR, CASP3, COBLL1, EEF2K, FBXO28, FGFR1OP, LRP12, MDM4, PAIP2, PARM1, PDE5A, PIAS2, SAR1B, SCD, SLC44A1, TIMM17A, TSPYL1, UBXN7, YES1, ZBTB37, ZNF587) through their 
intersection. These may be the key target gene of miR-382-5P regulating ovarian cancer. Therefore, this study will improve the research direction of miR-382$5 \mathrm{P}$ target gene in ovarian cancer.

In order to further study the function of miR-382-5P, 116 target genes were selected to annotate GO function. It was found that microRNAs participate in biological processes such as peptide chain-serine phosphorylation, lipopolysaccharide-mediated signaling pathway, apoptosis, etc. Molecular functions such as serine/threonine kinase activity, ATP binding, siRNA binding and so on. Cell composition such as cytoplasm, cell membrane and cell nucleus. It is suggested that miR-382-5P may be involved in the regulation of these biological processes in ovarian cancer. (As shown in Table 2-4) At the same time, 116 target genes were enriched in KEGG pathway. It was found that they were significantly enriched in microRNAs in cancer, MAPK signaling pathway, Hepatitis B, FoxO signaling pathway, Non-small cell lung cancer, Apoptosis, Colorectal cancer, Oxytocin signaling pathway (Table 6). In recent years, related literatures have reported that miR-382-5P participates in related functions. In breast cancer ${ }^{[4]} \rrbracket$ miR-382-5P activates Ras/ERK signaling pathway by targeting RERG, and promotes the biological behavior of tumor cells, such as viability, clonogenicity, survival, migration and invasion. In primary myelofibrosis ${ }^{[9]}$, miR-382-5P downregulates SOD2 by targeting, resulting in excessive accumulation of ROS, which ultimately leads to DNA oxidative stress. In colorectal cancer ${ }^{\text {[22] }}$ and osteosarcoma ${ }^{[16]}$, miR-382 can enhance the ability of cisplatin-induced apoptosis and promote the sensitivity of cells to cisplatin by targeting down-regulation of HIPK3. In primary hepatocellular carcinoma ${ }^{[11]}$, hepatitis B virus infection can induce the up-regulation of miR-382-5P, while miR-382-5P promotes cell metastasis and invasion by targeting down-regulation of DLC-1. In non-small cell lung cancer ${ }^{[15]}$, miR-382 inhibits cell proliferation, invasion and metastasis by targeting down-regulation of SETD8. In addition, Tan et al. ${ }^{[20]}$ found that miR-382 was down-regulated in ovarian cancer cell line SKOV3 and ovarian cancer tissues, which mediated the biological behavior of cancer cells such as metastasis, invasion and EMT by targeting ROR1. In our previous in vitro experiments ${ }^{[26]}$, we found that the expression of miR-382-5p in ovarian cancer drug-resistant cell line SKOV3-DDP was up-regulated compared with that in parent cell line SKOV3. At the same time, we found that the expression of miR-382-5p in ovarian cancer multidrug-resistant tissue was higher than that in sensitive tissue. Therefore, we speculate that there is a close relationship between miR-382-5p and drug resistance of ovarian cancer. Based on the above analysis, the related literatures reported above provide some credibility for viral hepatitis B, non-cellular lung cancer, FoxO pathway, apoptosis, colorectal cancer enriched by KEGG pathway in this study. It also shows that miR-382-5P participates in biological processes such as cell activity, proliferation, clonogenicity, survival, migration, invasion, DNA oxidative stress, drug resistance and EMT.

Chi-square test results in this study showed that the expression of miR-382-5p was positively correlated with the degree of drug resistance in ovarian cancer patients, and the difference was statistically significant $(\mathrm{P}<0.001)$. The sensitivity, specificity, positive likelihood ratio, negative likelihood ratio, positive predictive value, negative predictive value and Yoden index of miR-382-5p in the diagnosis of multidrug resistance in ovarian cancer were $56 \%$, $91 \%, 6.22,48 \%$, $93 \%, 48 \%$ and $47 \%$ respectively. The area under ROC curve was $70.5 \%$, The difference was statistically significant $(P<0.01)$. The above results showed that miR-382-5p was used to judge the resistance of ovarian cancer. It has good accuracy and can be used as an experimental diagnostic method for drug resistance of ovarian cancer.

The results of Kaplan-Meier analysis showed that residual lesions less than $1 \mathrm{~cm}$, no recurrence, cisplatin sensitivity, better therapeutic response, low expression of miR-382-5P were positively correlated with PFS in patients with ovarian cancer, the difference was statistically significant $(\mathrm{P}<0.05)$. Low expression of miR-382-5P, better therapeutic response, sensitivity to cisplatin and no recurrence were positively correlated with OS in patients with ovarian cancer, and the difference was statistically significant $(P<0.05)$. Therefore, we speculate that patients with ovarian cancer with high expression of miR-382-5P are closely related to their poor prognosis.

In Cox univariate analysis model, in order to prevent missing important indicators, the range of $\mathrm{P}$ value was broadened to 0.15 . The results showed that the factors of stage II, III, IV, drug resistance, high level of miR-382-5P, recurrence, disease stability and disease progression in FIGO stage were independent risk factors affecting the prognosis of ovarian cancer. Residual lesions ${ }^{[30]}$, histological grade and pathological type ${ }^{[31]}$ were correlated with their prognosis. The above factors were included in Cox multivariate analysis model. The results showed that only drug resistance was included in the model, and showed that drug resistance was an independent risk factor affecting the prognosis of ovarian cancer patients , the difference was statistically significant $(P<0.05)$. Therefore, we speculate that drug resistance is an independent risk for the prognosis of ovarian cancer patients.

The results of TCGA database data analysis showed no correlation between the expression of miR-382-5p and age, unilateral and bilateral, histological grade, FIGO stage, lymph node metastasis, vascular invasion, drug resistance, recurrence, survival status, treatment response, residual tumor size, tumor diameter, etc. There was no significant difference $(P>0.05)$. The clinical data collected in this study concluded that high level of miR-382-5p was closely related to the degree of drug resistance and poor prognosis of ovarian cancer patients. It was inconsistent with the conclusion of TCGA data analysis. The factors considered included regional differences and missing data of the study population. In addition, recent studies have reported that there is a clinical correlation between miR-382-5p and tumors. Ho et al. ${ }^{[4]}$ found that miR-382-5p is highly expressed in breast cancer and positively correlated with its adverse prognosis. It can be used as a prognostic and diagnostic index. Du et al. ${ }^{[11]}$ found that miR-382-5p is highly expressed in primary hepatocellular carcinoma of hepatitis B virus and can be used as a diagnostic index. It has also been reported that the expression of miR-382 is low in osteosarcoma ${ }^{[16]}$ and colorectal cancer ${ }^{[22]}$, and is positively correlated with its adverse prognosis and drug resistance, which can be used as an index of drug resistance and prognosis. This study reported that the expression of miR-382 was low in ovarian cancer tissues ${ }^{[20]}$, while the analysis of our study found that miR-382-5p was highly expressed in resistant tissues of ovarian cancer than sensitive tissues. At the same time, our previous study ${ }^{[26]}$ also found that the expression of miR-382-5p was higher in ovarian cancer resistant cell lines than in parental cell lines. The results of the related literatures reported above provide theoretical support for the analysis of this study that miR-382-5p can be used as a marker of multidrug resistance and prognosis in ovarian cancer.

In conclusion, miR-382-5p can be used as a marker of drug resistance and prognosis in the diagnosis of multidrug-resistant ovarian cancer, and it may be a prognostic factor in multidrug-resistant ovarian cancer patients. 
At present, many studies have shown that the expression of microRNA-382-5P is abnormal in many diseases, indicating that it plays an important role in the occurrence and development of diseases. The mechanism may be that microRNA regulates the activation or suppression of signaling pathways by targeting one or more target genes. microRNA network diagram and signal pathway network diagram are intricate and intermingled, which promote the occurrence of many abnormal pathological processes, leading to the occurrence and development of diseases. The study of mechanism is still an important problem to overcome cancer. Previous studies ${ }^{[26]}$ have confirmed that HIPK3 is a target gene of miR-382-5P in ovarian cancer. miR-382-5P promotes cell resistance to cisplatin by targeting down-regulation of HIPK3. Therefore, this study used cBioPort database to download HIPK3-related genes and enrich them in KEGG pathway. Selected items with $\mathrm{P}<0.05$, we found that HIPK3-related genes are significantly enriched in Signaling pathways regulating pluripotency of stem cells, Platelet activation and Ras signaling pathway. $(P<0.05)$ (Table 5)And Ho et al. ${ }^{[4]}$ found that in breast cancer, miR-382-5P activates Ras/ERK signaling pathway by targeting RERG, and promotes the biological behavior of tumor cells, such as viability, clonogenicity, survival, migration and invasion. Ras signaling pathway is one of the apoptotic signaling pathways. Therefore, we obtained Ras pathway by combining KEGG analysis of HIPK3-related gene and KEGG analysis of miR-382-5P target gene. It is speculated that in ovarian cancer, miR-382-5P may regulate Ras pathway by targeting HIPK3, leading to cell resistance to cisplatin.

By analyzing the correlation between the expression level of miR-382-5p and its clinicopathological parameters in ovarian cancer, and with the help of the expansion of bioinformatics knowledge, this study will help us to understand more about the mechanism of miR-382-5p mediating various biological behaviors, and provide a certain direction and theoretical basis for subsequent experimental research. However, there are still some limitations in this study. In ovarian cancer, whether miR-382-5p regulates Ras pathway by targeting HIPK3 has not yet been clarified, and the target genes and their functions predicted by bioinformatics have not yet been verified. Further experimental verification is needed to ensure the accuracy of the results and provide dawn for targeted treatment of multidrug-resistant ovarian cancer patients.

\section{Declarations}

Ethics approval

The research on human genetic resources materials in the "Analysis of the expression and clinical significance of miR-382-5P in ovarian cancer based on biological information" by Wu Peiyang was reviewed by the Ethics Committee of the affiliated tumor hospital of Guangxi medical university, and it was considered that the study met the requirements of medical ethics.

Consent for publication

I will use my institutional consent form.

Availability of data and materials

Data availability was stated in the manuscript.

Competing interests

The authors declare that they have no competing interests.

Funding

This work was supported by grants from the National Natural Science Foundation of China (Grant No. 81572579, No. 81660430). The funder had no preparation of the manuscript. role in study design, data collection and analysis, decision to publish, or preparation of the manuscript.

Authors' contributions

Wu Peiyang queried, analyzed and interpreted the datas regarding the ovarian cancer, was a major contributor in writing the manuscript. Li Li provided modification suggestions, was the head of the fund.

Acknowledgements

Not applicable

Department of Gynecologic Oncology, Affifiliated Tumor Hospital of Guangx Medical University, 71 Hedi Road, Nanning, Guangxi, PR China

* 2019. Search keywords such as "ovarian cancer or ovarian Correspondence: Li Li, Department of Gynecologic Oncology, Affifiliated Tumor Hospital of Guangxi Medical University, 71 Hedi Road, Nanning, Guangxi 530021, PR China (e-mail: lili@gxmu.edu.cn).

\section{References}

[1] Siegel RL, Miller KD, Jemal A.Cancer statistics, 2019.CA Cancer J Clin 2019;69(1):7-34.http://dx.doi.org/10.3322/caac.21551

[2] Meghani K, Fuchs W, Detappe A, Drane P, Gogola E, Rottenberg S, Jonkers J, Matulonis U, Swisher EM, Konstantinopoulos PA, Chowdhury D.Multifaceted Impact of MicroRNA 493-5p on Genome-Stabilizing Pathways Induces Platinum and PARP Inhibitor Resistance in BRCA2-Mutated Carcinomas.Cell Rep 2018;23(1):100-111.http://dx.doi.org/10.1016/j.celrep.2018.03.038

Page $7 / 31$ 
[3] Li B, Wang F, Li X, Sun S, Shen Y, Yang H.Hsa_circ_0008309 May Be a Potential Biomarker for Oral Squamous Cell Carcinoma.Disease markers 2018;2018:7496890.http://dx.doi.org/10.1155/2018/7496890

[4] Ho JY, Hsu RJ, Liu JM, Chen SC, Liao GS, Gao HW, Yu CP.MicroRNA-382-5p aggravates breast cancer progression by regulating the RERG/Ras/ERK signaling axis.Oncotarget 2017;8(14):22443-22459.http://dx.doi.org/10.18632/oncotarget.12338

[5] He Q, Zhao L, Liu X, Zheng J, Liu Y, Liu L, Ma J, Cai H, Li Z, Xue Y.MOV10 binding circ-DICER1 regulates the angiogenesis of glioma via miR-103a3p/miR-382-5p mediated ZIC4 expression change.Journal of experimental \& clinical cancer research : CR 2019;38(1):9.http://dx.doi.org/10.1186/s13046-0180990-1

[6] Wang J, Chen C, Yan X, Wang P.The role of miR-382-5p in glioma cell proliferation, migration and invasion.Onco Targets Ther 2019;12:49935002.http://dx.doi.org/10.2147/ott.S196322

[7] Liu D, Zhong L, Yuan Z, Yao J, Zhong P, Liu J, Yao S, Zhao Y, Liu L, Chen M, Li L, Liu B.miR-382-5p modulates the ATRA-induced differentiation of acute promyelocytic leukemia by targeting tumor suppressor PTEN.Cellular signalling 2019;54:1-9.http://dx.doi.org/10.1016/j.cellsig.2018.11.012

[8] Zini R, Rossi C, Norfo R, Pennucci V, Barbieri G, Ruberti S, Rontauroli S, Salati S, Bianchi E, Manfredini R.miR-382-5p Controls Hematopoietic Stem Cell Differentiation Through the Downregulation of MXD1.Stem cells and development 2016;25(19):1433-1443.http://dx.doi.org/10.1089/scd.2016.0150

[9] Rossi C, Zini R, Rontauroli S, Ruberti S, Prudente Z, Barbieri G, Bianchi E, Salati S, Genovese E, Bartalucci N, Guglielmelli P, Tagliafico E, Rosti V, Barosi G, Vannucchi AM, Manfredini R.Role of TGF-beta1/miR-382-5p/SOD2 axis in the induction of oxidative stress in CD34+ cells from primary myelofibrosis.Molecular oncology 2018;12(12):2102-2123.http://dx.doi.org/10.1002/1878-0261.12387

[10] Lin CA, Duan KY, Wang XW, Zhang ZS.Study on the role of Hsa-miR-382-5p in epidural fibrosis.European review for medical and pharmacological sciences 2018;22(12):3663-3668.http://dx.doi.org/10.26355/eurrev_201806_15244

[11] Du J, Bai F, Zhao P, Li X, Li X, Gao L, Ma C, Liang X.Hepatitis B core protein promotes liver cancer metastasis through miR-382-5p/DLC-1

axis.Biochimica et biophysica acta Molecular cell research 2018;1865(1):1-11.http://dx.doi.org/10.1016/j.bbamcr.2017.09.020

[12] Hu YW, Zhao JY, Li SF, Huang JL, Qiu YR, Ma X, Wu SG, Chen ZP, Hu YR, Yang JY, Wang YC, Gao JJ, Sha YH, Zheng L, Wang Q.RP5-833A20.1/miR-382$5 \mathrm{p} / \mathrm{NFIA-dependent} \mathrm{signal} \mathrm{transduction} \mathrm{pathway} \mathrm{contributes} \mathrm{to} \mathrm{the} \mathrm{regulation} \mathrm{of} \mathrm{cholesterol} \mathrm{homeostasis} \mathrm{and} \mathrm{inflammatory} \mathrm{reaction.Arteriosclerosis,}$ thrombosis, and vascular biology 2015;35(1):87-101.http://dx.doi.org/10.1161/atvbaha.114.304296

[13] Han MS, Lee JM, Kim SN, Kim JH, Kim HS.Human Papillomavirus 16 Oncoproteins Downregulate the Expression of miR-148a-3p, miR-190a-5p, and miR-199b-5p in Cervical Cancer.BioMed research international 2018;2018:1942867.http://dx.doi.org/10.1155/2018/1942867

[14] Chen D, Zhang Y, Lin Y, Shen F, Zhang Z, Zhou J.MicroRNA-382 inhibits cancer cell growth and metastasis in NSCLC via targeting LMO3.Experimental and therapeutic medicine 2019;17(4):2417-2424.http://dx.doi.org/10.3892/etm.2019.7271

[15] Chen T, Ren H, Thakur A, Yang T, Li Y, Zhang S, Wang T, Chen M.miR-382 inhibits tumor progression by targeting SETD8 in non-small cell lung cancer.Biomedicine \& pharmacotherapy = Biomedecine \& pharmacotherapie 2017;86:248-253.http://dx.doi.org/10.1016/j.biopha.2016.12.007

[16] Xu M, Jin H, Xu CX, Sun B, Mao Z, Bi WZ, Wang Y.miR-382 inhibits tumor growth and enhance chemosensitivity in osteosarcoma.Oncotarget 2014;5(19):9472-9483.http://dx.doi.org/10.18632/oncotarget.2418

[17] Wang S, Wen X, Han XR, Wang YJ, Shen M, Fan SH, Zhuang J, Zhang ZF, Shan Q, Li MQ, Hu B, Sun CH, Wu DM, Lu J, Zheng YL.Repression of microRNA-382 inhibits glomerular mesangial cell proliferation and extracellular matrix accumulation via Fox01 in mice with diabetic nephropathy.Cell proliferation 2018;51(5):e12462.http://dx.doi.org/10.1111/cpr.12462

[18] Fang Y, Xie T, Xue N, Kuang Q, Wei Z, Liang M, Ding X.miR-382 Contributes to Renal Tubulointerstitial Fibrosis by Downregulating HSPD1.Oxidative medicine and cellular longevity 2017;2017:4708516.http://dx.doi.org/10.1155/2017/4708516

[19] Zhang W, Liu J, Qiu J, Fu X, Tang Q, Yang F, Zhao Z, Wang H.MicroRNA-382 inhibits prostate cancer cell proliferation and metastasis through targeting COUP-TFII.Oncology reports 2016;36(6):3707-3715.http://dx.doi.org/10.3892/or.2016.5141

[20] Tan H, He Q, Gong G, Wang Y, Li J, Wang J, Zhu D, Wu X.miR-382 inhibits migration and invasion by targeting ROR1 through regulating EMT in ovarian cancer.International journal of oncology 2016;48(1):181-190.http://dx.doi.org/10.3892/ijo.2015.3241

[21] Ren Y, Zhang H, Jiang P.MicroRNA-382 inhibits cell growth and migration in colorectal cancer by targeting SP1.Biological research 2018;51(1):51.http://dx.doi.org/10.1186/s40659-018-0200-9

[22] Yao H, Xia D, Li ZL, Ren L, Wang MM, Chen WS, Hu ZC, Yi GP, Xu L.MiR-382 functions as tumor suppressor and chemosensitizer in colorectal cancer.Bioscience reports 2019;39(8).http://dx.doi.org/10.1042/bsr20180441

[23] Li D, Li P, Guo Z, Wang H, Pan W.Downregulation of miR-382 by propranolol inhibits the progression of infantile hemangioma via the PTEN-mediated AKT/mTOR pathway.International journal of molecular medicine 2017;39(3):757-763.http://dx.doi.org/10.3892/ijmm.2017.2863 
[24] Zhang S, Ge W, Zou G, Yu L, Zhu Y, Li Q, Zhang Y, Wang Z, Xu T.MiR-382 targets GOLM1 to inhibit metastasis of hepatocellular carcinoma and its down-regulation predicts a poor survival.American journal of cancer research 2018;8(1):120-131

[25] Mor E, Kano S, Colantuoni C, Sawa A, Navon R, Shomron N.MicroRNA-382 expression is elevated in the olfactory neuroepithelium of schizophrenia patients.Neurobiology of disease 2013;55:1-10.http://dx.doi.org/10.1016/j.nbd.2013.03.011

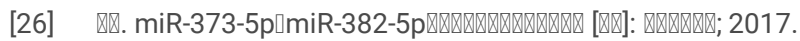

[27] Lee RC, Feinbaum RL, Ambros V.The C. elegans heterochronic gene lin-4 encodes small RNAs with antisense complementarity to lin-14.Cell 1993;75(5):843-854.http://dx.doi.org/10.1016/0092-8674(93)90529-y

[28] Mendell JT, Olson EN.MicroRNAs in stress signaling and human disease.Cell 2012;148(6):1172-1187.http://dx.doi.org/10.1016/j.cell.2012.02.005

[29] Di Leva G, Croce CM.The Role of microRNAs in the Tumorigenesis of Ovarian Cancer.Frontiers in oncology

2013;3:153.http://dx.doi.org/10.3389/fonc.2013.00153

[30] Kim KH, Choi KU, Kim A, Lee SJ, Lee JH, Suh DS, Kwon BS, Hwang C.PD-L1 expression on stromal tumor-infiltrating lymphocytes is a favorable prognostic factor in ovarian serous carcinoma.Journal of ovarian research 2019;12(1):56.http://dx.doi.org/10.1186/s13048-019-0526-0

[31] Chudecka-Glaz A, Cymbaluk-Ploska A, Wezowska M, Menkiszak J.Could HE4 level measurements during first-line chemotherapy predict response to treatment among ovarian cancer patients?PloS one 2018;13(3):e0194270.http://dx.doi.org/10.1371/journal.pone.0194270

\section{Tables}

Table 1 Diseases regulated by miR-382-5P

\begin{tabular}{|c|c|c|c|c|}
\hline microRNA & Related diseases & $\begin{array}{l}\text { Expression } \\
\text { level }\end{array}$ & Target gene & Biological process \\
\hline $\begin{array}{l}\operatorname{miR}-382- \\
5 P\end{array}$ & glioma & $\downarrow$ & $\mathrm{ZIC} 4^{[5]}, \mathrm{YBX} 1^{[6]}$ & $\begin{array}{l}\text { Cell viability, migration, angiogenesis, EMT, proliferation and } \\
\text { invasion }\end{array}$ \\
\hline $\operatorname{miR}_{5 P}$ & $\begin{array}{l}\text { acute promyelocytic } \\
\text { leukemia }\end{array}$ & $\uparrow$ & $\mathrm{PTEM}^{[7]}, \mathrm{MXD} 1^{[8]}$ & hematopoietic stem cell differentiation \\
\hline $\begin{array}{l}\operatorname{miR}-382- \\
5 P\end{array}$ & primary myelofibrosis & $\uparrow$ & $\operatorname{SOD} 2^{[9]}$ & DNA oxidative stress and inflammation \\
\hline $\begin{array}{l}\operatorname{miR}_{5 \mathrm{P}}-382- \\
\end{array}$ & epidural fibrosis & $\uparrow$ & CollagenIA $1^{[10]}$ & epidural fibrosis \\
\hline $\begin{array}{l}\operatorname{miR}-382- \\
5 P\end{array}$ & primary liver cancer & $\uparrow$ & $\mathrm{DLC}^{[11]}$ & migration \\
\hline $\begin{array}{l}\operatorname{miR}-382- \\
5 P\end{array}$ & Breast cancer & $\uparrow$ & $\mathrm{RERG}^{[4]}$ & cell viability, colony-formation, migration, invasion, proliferation \\
\hline $\begin{array}{l}\operatorname{miR}-382- \\
5 P\end{array}$ & atherosclerosis & $\uparrow$ & NFIA ${ }^{[12]}$ & cholesterol homeostasis,inflammatory reaction \\
\hline miR-382 & non-small cell lung cancer & $\downarrow$ & $\mathrm{LMO3}^{[14]}, \mathrm{SETD}^{[15]}$ & proliferation, metastasis, invasion \\
\hline miR-382 & osteosarcoma & $\downarrow$ & $\mathrm{KLF} 12, \mathrm{HIPK}^{[16]}$ & growth,drug resistance, prognosis \\
\hline miR-382 & schizophrenia & $\uparrow$ & FGFR1,SPRY[25] & abnormal brain development and function \\
\hline miR-382 & diabetic nephropathy & $\uparrow$ & FoxO1 ${ }^{[17]}$ & $\begin{array}{l}\text { glomerular mesangial cell proliferation, extracelluar matrix } \\
\text { accumulation }\end{array}$ \\
\hline miR-382 & IgA nephropathy & $\uparrow$ & HSPD $1^{[18]}$ & renal tubulointerstit-ial fibrosis \\
\hline miR-382 & prostate cancer & $\downarrow$ & COUP-TFII ${ }^{[19]}$ & metastasis, proliferation, invasion \\
\hline miR-382 & ovarian cacner & $\downarrow$ & $\mathrm{ROR} 1^{[20]}$ & proliferation, invasion, metastasis, EMT \\
\hline miR-382 & colorectal cancer & $\downarrow$ & $\begin{array}{l}\mathrm{SP} 1^{[21]}, \mathrm{KLF} 12, \\
\mathrm{HIPK}^{[22]}\end{array}$ & proliferation, drug resistance, migration \\
\hline miR-382 & primary liver cancer & $\downarrow$ & GOLM1 ${ }^{[24]}$ & migration, invasion, prognosis \\
\hline miR-382 & infantile hemangioma & $\uparrow$ & PTEN ${ }^{[23]}$ & proliferation,metastasis, apotosis \\
\hline
\end{tabular}




\begin{tabular}{|c|c|c|c|}
\hline Category & Term & P Va & Genes \\
\hline GOTERM_BP_DIRECT & G0:0018105 peptidyl-serine phosphorylation & 0.005415 & $\begin{array}{l}\text { PRKCA, CLSPN, SGK1, TGFBR1, } \\
\text { DYRK1A }\end{array}$ \\
\hline GOTERM_BP_DIRECT & Go:1900119 positive regulation of execution phase of apoptosis & 0.039281 & DLC1, TP53 \\
\hline GOTERM_BP_DIRECT & $\begin{array}{l}\text { GO:0031666 positive regulation of lipopolysaccharide-mediated } \\
\text { signaling pathway }\end{array}$ & 0.045678 & PRKCA, SASH1 \\
\hline GOTERM_BP_DIRECT & G0:0043066 negative regulation of apoptotic process & 0.047713 & $\begin{array}{l}\text { CASP3, TP53, PRKAA1, MDM4, } \\
\text { CLN8 }\end{array}$ \\
\hline
\end{tabular}

Table 3 miR-382-5p Target Gene-Cell Composition

\begin{tabular}{|llll|}
\hline Category & Term & P Value & Genes \\
\hline GOTERM_MF_DIRECT & $\begin{array}{l}\text { G0:0004674 protein } \\
\text { serine/threonine kinase activity }\end{array}$ & 0.002105 & PRKCA, SGK1, SLK, AAK1, DYRK1A, EEF2K, PRKAA1 \\
GOTERM_MF_DIRECT & G0:0005524 ATP binding & 0.005857 & $\begin{array}{l}\text { PRKCA, DHX8, SGK1, TGFBR1, DICER1, TP53, TRIB1, ATP6V1A, GLUL, SLK, } \\
\text { AAK1, DYRK1A, EEF2K, PRKAA1, DDX21, YES1 }\end{array}$ \\
\hline GOTERM_MF_DIRECT & G0:0035197 siRNA binding & 0.044334 & DICER1, MECP2 \\
\hline
\end{tabular}

Table4 miR-382-5p Target Gene-Molecular Function

\begin{tabular}{|llll|}
\hline Category & Term & P Value & Genes \\
\hline GOTERM_MF_DIRECT & $\begin{array}{l}\text { G0:0004674 protein } \\
\text { serine/threonine kinase activity }\end{array}$ & 0.002105 & PRKCA, SGK1, SLK, AAK1, DYRK1A, EEF2K, PRKAA1 \\
\hline GOTERM_MF_DIRECT & G0:0005524 ATP binding & 0.005857 & $\begin{array}{l}\text { PRKCA, DHX8, SGK1, TGFBR1, DICER1, TP53, TRIB1, ATP6V1A, GLUL, SLK, } \\
\text { AAK1, DYRK1A, EEF2K, PRKAA1, DDX21, YES1 }\end{array}$ \\
\hline GOTERM_MF_DIRECT & G0:0035197 siRNA binding & 0.044334 & DICER1, MECP2 \\
\hline
\end{tabular}

Table 5 KEGG analysis of HIPK3-related genes

\begin{tabular}{|llll|}
\hline Category & Term & P Value & Genes \\
\hline KEGG_PATHWAY & $\begin{array}{l}\text { hsa04550:Signaling pathways regulating } \\
\text { pluripotency of stem cells }\end{array}$ & 0.001302 & $\begin{array}{l}\text { JARID2, PIK3CB, IL6ST, FZD2, ESX1, FZD6, PCGF5, PCGF2, HAND1, JAK1, } \\
\text { JAK2, DUSP9, TCF3, PIK3R1, ACVR1 }\end{array}$ \\
\hline KEGG_PATHWAY & hsa04611:Platelet activation & 0.034128 & $\begin{array}{l}\text { GNAQ, P2RX1, PIK3CB, TLN2, PPP1R12A, STIM1, PLA2G4F, GNAS, } \\
\text { ARHGEF12, PIK3R1, ITGA2B }\end{array}$ \\
\hline KEGG_PATHWAY & hsa04014:Ras signaling pathway & 0.036043 & $\begin{array}{l}\text { FGF19, PIK3CB, EFNA3, FGF11, GNG12, RGL2, PLCG1, RRAS2, PLA2G12B, } \\
\text { RAB5A, PLA2G6, PLA2G4F, GNB3, EFNA4, PIK3R1, FGF4 }\end{array}$ \\
\hline
\end{tabular}

Table 6 KEGG analysis of miR-382-5p target gene 


\begin{tabular}{|llll|}
\hline Category & Term & P Value & Genes \\
\hline KEGG_PATHWAY & microRNAs in cancer & 0.001 & PRKCA, NRAS, CASP3, DICER1, TP53, MDM4 \\
\hline KEGG_PATHWAY & MAPK signaling pathway & 0.00238 & PRKCA, NRAS, CASP3, TGFBR1, TP53, RAP1A, TAB2 \\
\hline KEGG_PATHWAY & Hepatitis B & 0.00796 & PRKCA, NRAS, CASP3, TGFBR1, TP53 \\
\hline KEGG_PATHWAY & FoxO signaling pathway & 0.03679 & NRAS, SGK1, TGFBR1, PRKAA1 \\
KEGG_PATHWAY & Non-small cell lung cancer & 0.04057 & PRKCA, NRAS, TP53 \\
\hline KEGG_PATHWAY & Apoptosis & 0.04187 & CASP3, XIAP, TP53 \\
\hline KEGG_PATHWAY & Colorectal cancer & 0.04587 & CASP3, TGFBR1, TP53 \\
\hline KEGG_PATHWAY & Oxytocin signaling pathway & 0.04903 & PRKCA, NRAS, EEF2K, PRKAA1 \\
\hline
\end{tabular}

Table 7 Correlation between miR-382-5p and its clinicopathological parameters in ovarian cancer (TCGA) 


\begin{tabular}{|c|c|c|c|c|c|}
\hline \multirow[t]{2}{*}{ Parameters } & \multirow[t]{2}{*}{ total } & \multicolumn{3}{|c|}{ expresion level of } & \multirow[t]{2}{*}{$P$ value } \\
\hline & & low & high & & \\
\hline \multicolumn{6}{|l|}{ Age } \\
\hline$\leq 60 y$ & 267 & 139 & 128 & 1.414 & 0.503 \\
\hline$\otimes 60 y$ & 219 & 104 & 115 & & \\
\hline defect & 3 & 1 & 2 & & \\
\hline \multicolumn{6}{|l|}{ Location } \\
\hline unilateral & 121 & 64 & 57 & 0.593 & 0.743 \\
\hline bilateral & 336 & 164 & 172 & & \\
\hline defect & 32 & 16 & 16 & & \\
\hline \multicolumn{6}{|l|}{ Grade } \\
\hline $\mathrm{G} 1 / \mathrm{GB} / \mathrm{GX}$ & 12 & 4 & 8 & 2.443 & 0.486 \\
\hline G2 & 56 & 25 & 31 & & \\
\hline G3 & 416 & 213 & 203 & & \\
\hline defect & 5 & 2 & 3 & & \\
\hline \multicolumn{6}{|l|}{ FIGO stage } \\
\hline $\mathrm{I} / \mathrm{II}$ & 28 & 18 & 10 & 2.666 & 0.246 \\
\hline III/IV & 454 & 222 & 232 & & \\
\hline defect & 7 & 4 & 3 & & \\
\hline \multicolumn{6}{|l|}{ Drug resistance } \\
\hline Yes & 18 & 9 & 9 & 0.393 & 0.822 \\
\hline No & 290 & 148 & 142 & & \\
\hline defect & 181 & 87 & 94 & & \\
\hline \multicolumn{6}{|l|}{ Recurrence } \\
\hline Yes & 232 & 113 & 119 & 0.481 & 0.786 \\
\hline No & 29 & 16 & 13 & & \\
\hline defect & 228 & 115 & 113 & & \\
\hline \multicolumn{6}{|l|}{ Survival status } \\
\hline survial & 179 & 89 & 90 & 0.422 & 1 \\
\hline death & 307 & 154 & 153 & & \\
\hline defect & 3 & 1 & 2 & & \\
\hline \multicolumn{6}{|l|}{ Therapeutic response } \\
\hline (Complete Remission ,CR) & 226 & 111 & 115 & 1.583 & 0.812 \\
\hline (Partial Remission ,PR) & 49 & 21 & 28 & & \\
\hline (Disease progress®PD囚 & 24 & 13 & 11 & & \\
\hline (Disease Stable ,SD) & 37 & 19 & 18 & & \\
\hline defect & 153 & 80 & 73 & & \\
\hline \multicolumn{6}{|l|}{ Lymph node metastasis } \\
\hline Yes & 114 & 53 & 61 & 3.276 & 0.194 \\
\hline No & 65 & 39 & 26 & & \\
\hline defect & 310 & 152 & 158 & & \\
\hline \multicolumn{6}{|l|}{ Vascular invasion } \\
\hline Yes & 73 & 36 & 37 & 0.535 & 0.765 \\
\hline
\end{tabular}

Page 12/31 


\begin{tabular}{|llllll|}
\hline No & 55 & 30 & 25 & & \\
\hline defect & 361 & 178 & 183 & & \\
\hline Residual tumor & & & & & \\
\hline No macroscopic disease & 92 & 55 & 37 & 5.653 & 0.227 \\
\hline $1-10 \mathrm{~mm}$ & 221 & 101 & 120 & & \\
$11-20 \mathrm{~mm}$ & 30 & 13 & 17 & & \\
\hline$>20 \mathrm{~mm}$ & 85 & 41 & 44 & & \\
\hline defect & 70 & 34 & 36 & & \\
\hline Tumor diameter & & & & & \\
\hline$\leq 0.8 \mathrm{~cm}$ & 198 & 95 & 103 & 2.839 & 0.242 \\
\hline $00.8 \mathrm{~cm}$ & 205 & 99 & 106 & & \\
\hline defect & 86 & 50 & 36 & & \\
\hline
\end{tabular}

Note: The size of residual lesions was divided by median.

Table 8 Correlation between miR-382-5p and clinicopathological parameters in ovarian cancer 


\begin{tabular}{|c|c|c|c|c|c|}
\hline \multirow[t]{2}{*}{ Parameters } & \multirow[t]{2}{*}{ total } & \multicolumn{2}{|c|}{$\begin{array}{l}\text { expresion level of } \\
\text { miR-382-5P }\end{array}$} & \multirow[t]{2}{*}{$\mathrm{X}^{2}$} & \multirow[t]{2}{*}{ P value } \\
\hline & & low & high & & \\
\hline \multicolumn{6}{|l|}{ Age } \\
\hline$\leqq 50 y$ & 40 & 11 & 29 & 0.396 & 0.529 \\
\hline$>50 y$ & 32 & 11 & 21 & & \\
\hline \multicolumn{6}{|l|}{ Grade } \\
\hline $\mathrm{G} 1$ & 60 & 18 & 42 & 0.329 & 1 \\
\hline $\mathrm{G} 2$ & 6 & 2 & 4 & & \\
\hline G3 & 6 & 2 & 4 & & \\
\hline \multicolumn{6}{|l|}{ FIGO stage } \\
\hline I & 9 & 3 & 6 & 0.488 & 1 \\
\hline II & 5 & 1 & 4 & & \\
\hline III & 52 & 16 & 36 & & \\
\hline IV & 6 & 2 & 4 & & \\
\hline \multicolumn{6}{|c|}{ Pathological type } \\
\hline serous & 40 & 16 & 24 & & \\
\hline mucous & 15 & 3 & 12 & 3.804 & 0.149 \\
\hline others & 17 & 3 & 14 & & \\
\hline \multicolumn{6}{|c|}{ Drug resistance } \\
\hline Yes & 30 & 2 & 28 & 13.831 & 0.0002 \\
\hline No & 42 & 20 & 22 & & \\
\hline \multicolumn{6}{|l|}{ Recurrence } \\
\hline Yes & 61 & 16 & 42 & 3.362 & 0.161 \\
\hline No & 6 & 3 & 3 & & \\
\hline defect & 5 & 3 & 2 & & \\
\hline \multicolumn{6}{|c|}{ Residual lesion } \\
\hline$\leq 1 \mathrm{~cm}$ & 53 & 16 & 37 & 0.013 & 0.91 \\
\hline$\nabla 1 \mathrm{~cm}$ & 19 & 6 & 13 & & \\
\hline \multicolumn{6}{|c|}{ Survival status } \\
\hline survival & 12 & 6 & 6 & 3.662 & 0.16 \\
\hline dead & 44 & 10 & 34 & & \\
\hline defect & 16 & 6 & 10 & & \\
\hline \multicolumn{6}{|l|}{ Response } \\
\hline $\mathrm{CR}$ & 44 & 17 & 27 & 4.299 & 0.342 \\
\hline PR & 2 & 0 & 2 & & \\
\hline SD & 5 & 0 & 5 & & \\
\hline PD & 11 & 2 & 9 & & \\
\hline defect & 10 & 3 & 7 & & \\
\hline
\end{tabular}

Note: The size of residual lesions was divided by median.

Table 9 Cox multivariate analysis of prognostic factors of ovarian cancer 


\begin{tabular}{|llll|}
\hline Parameter & P value & HR & 95\%Cl \\
\hline Drug resistance & & & \\
no & & 1 [reference] & \\
yes & 0.027 & 2.149 & $1.092-4.23$ \\
\hline
\end{tabular}

\section{Figures}

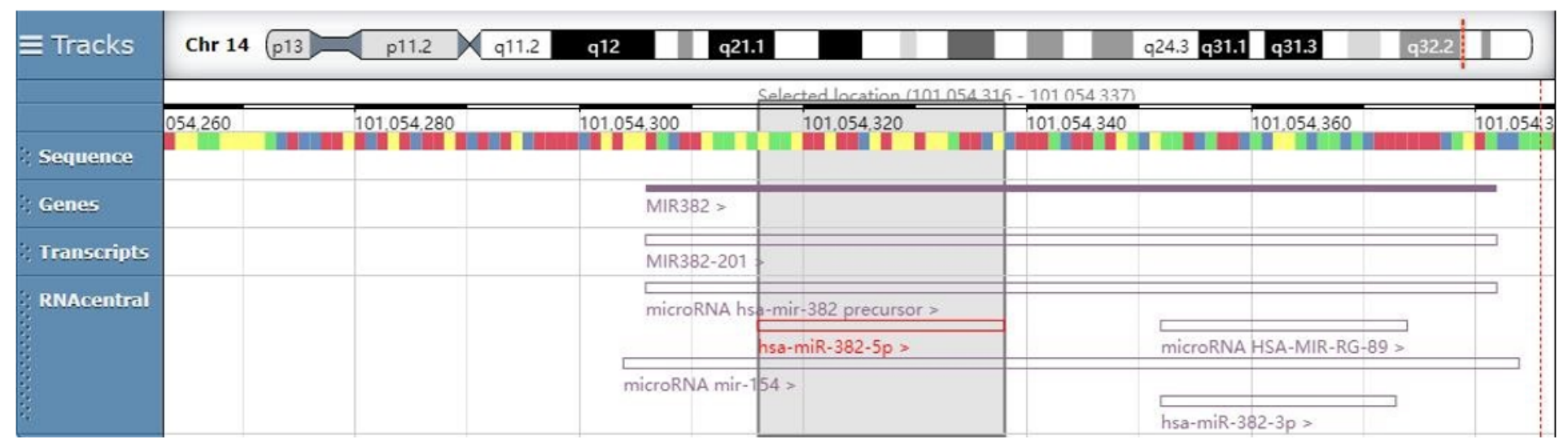

Figure 1

Basic information of miR-382-5P 


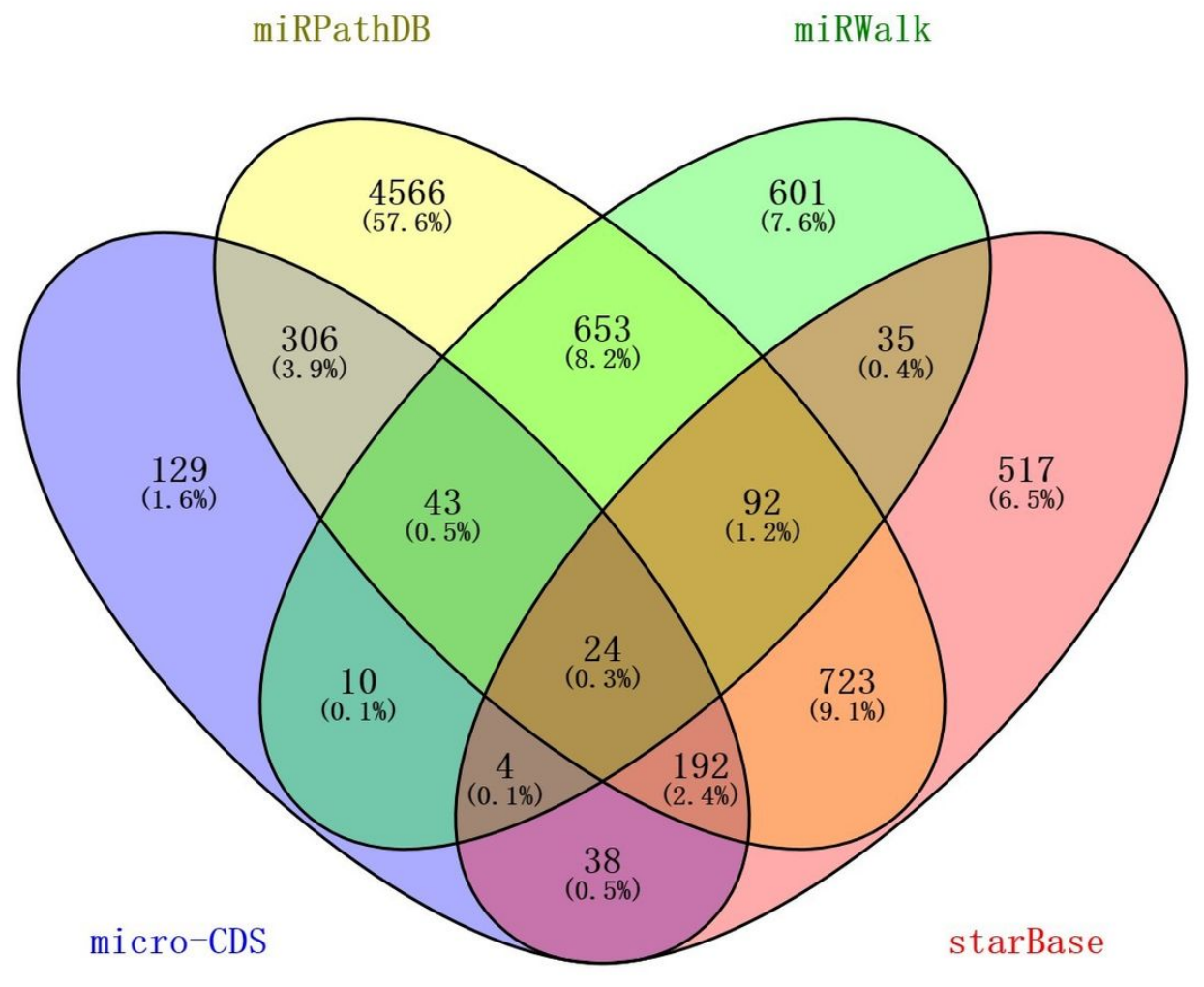

Figure 2

The intersection results of miRPathDB, DIANA, miRWalk and starBase 


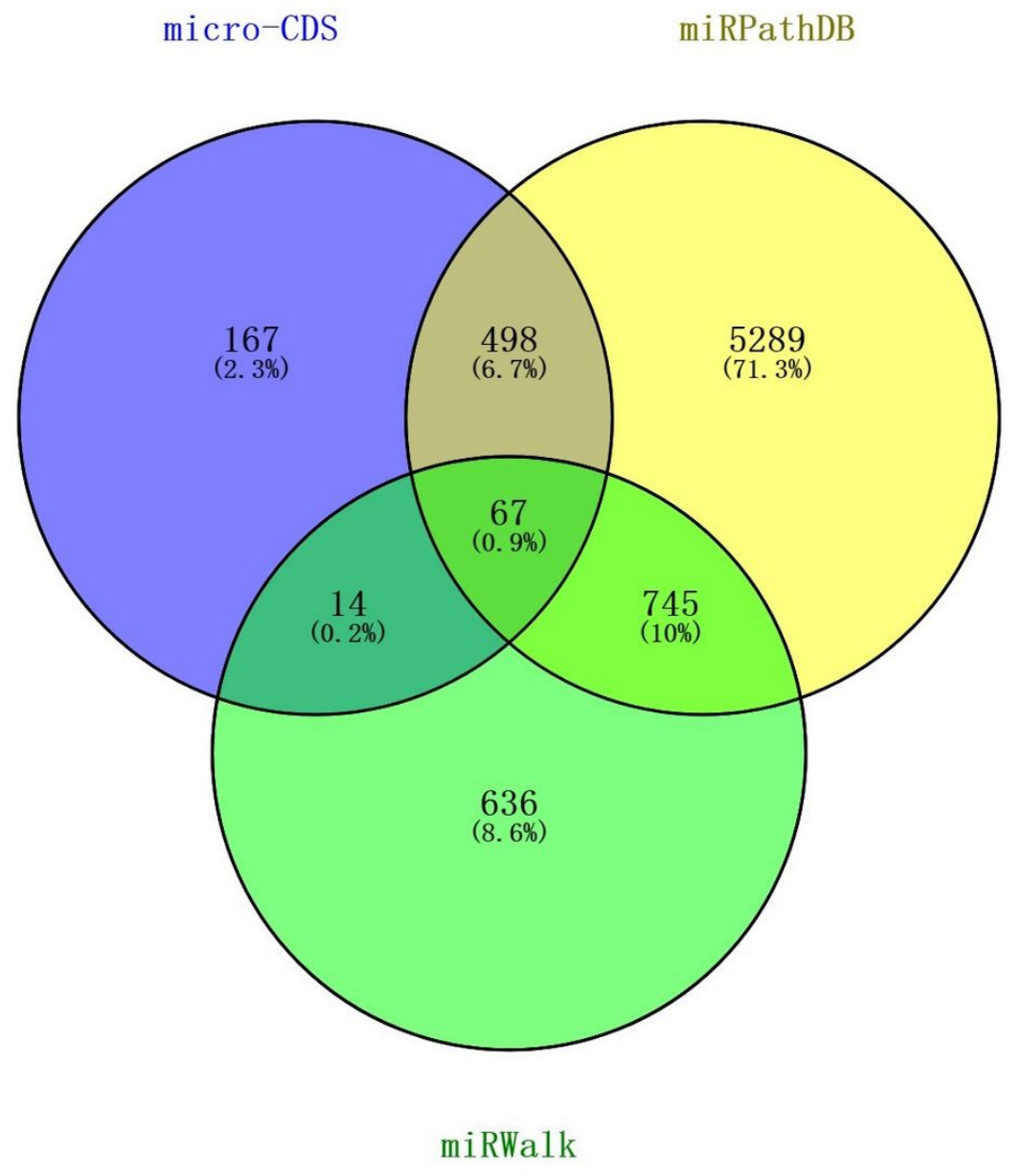

Figure 3

The intersection results of miRPathDB, DIANA and miRWalk 


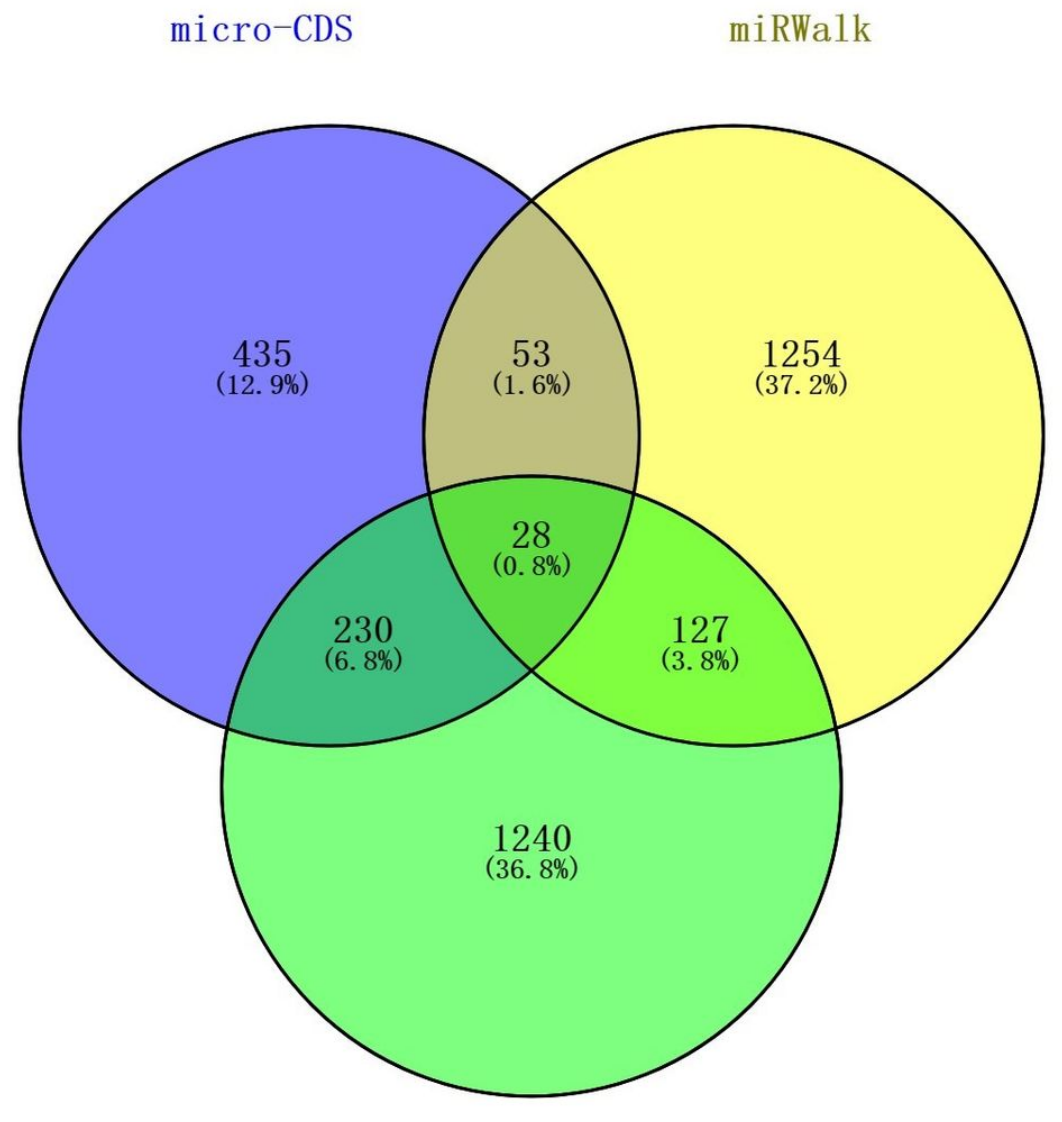

starBase

Figure 4

The intersection results of DIANA, miRWalk and starBase 


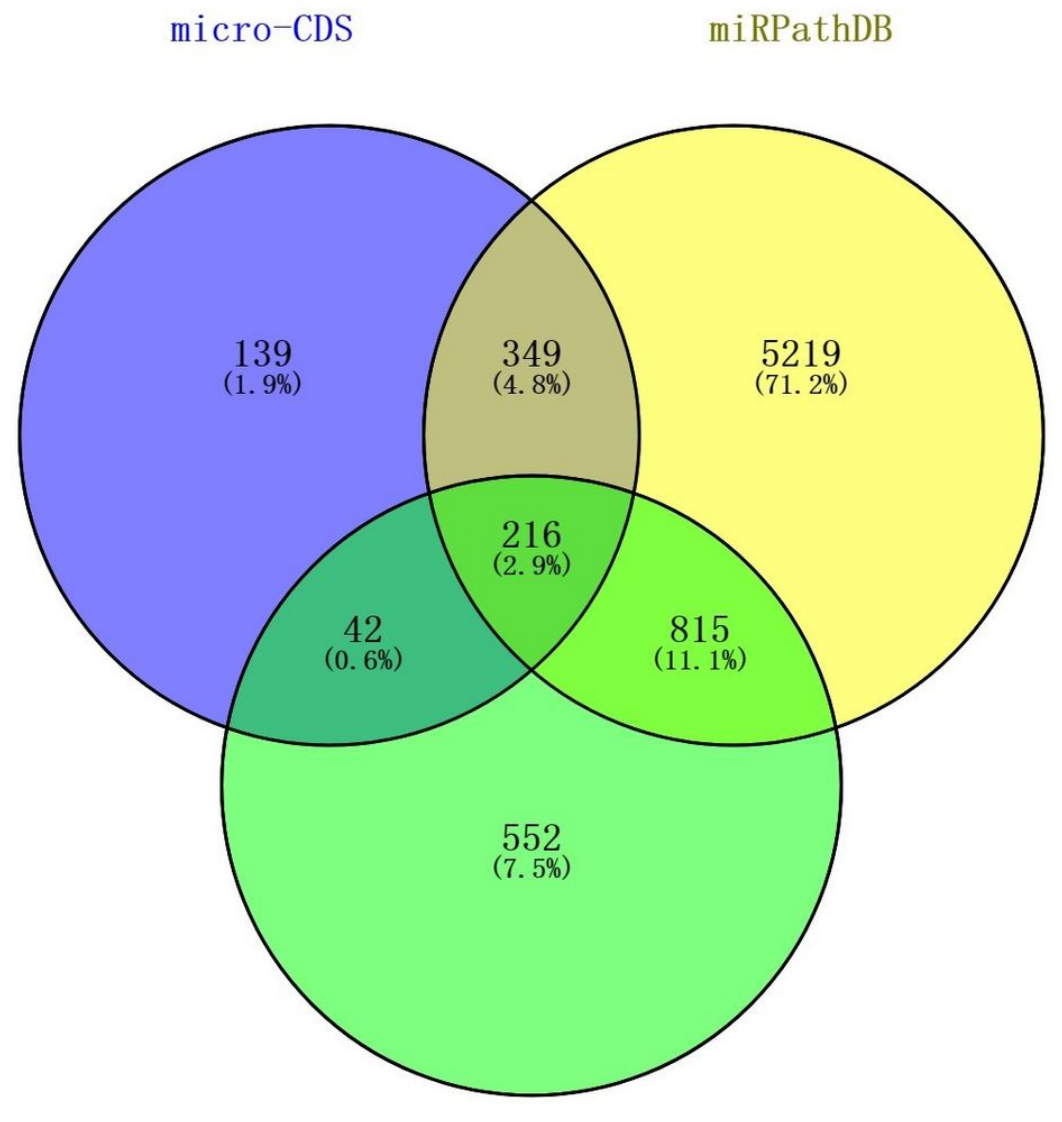

starBase

Figure 5

The intersection results of miRPathDB, DIANA and starBase 


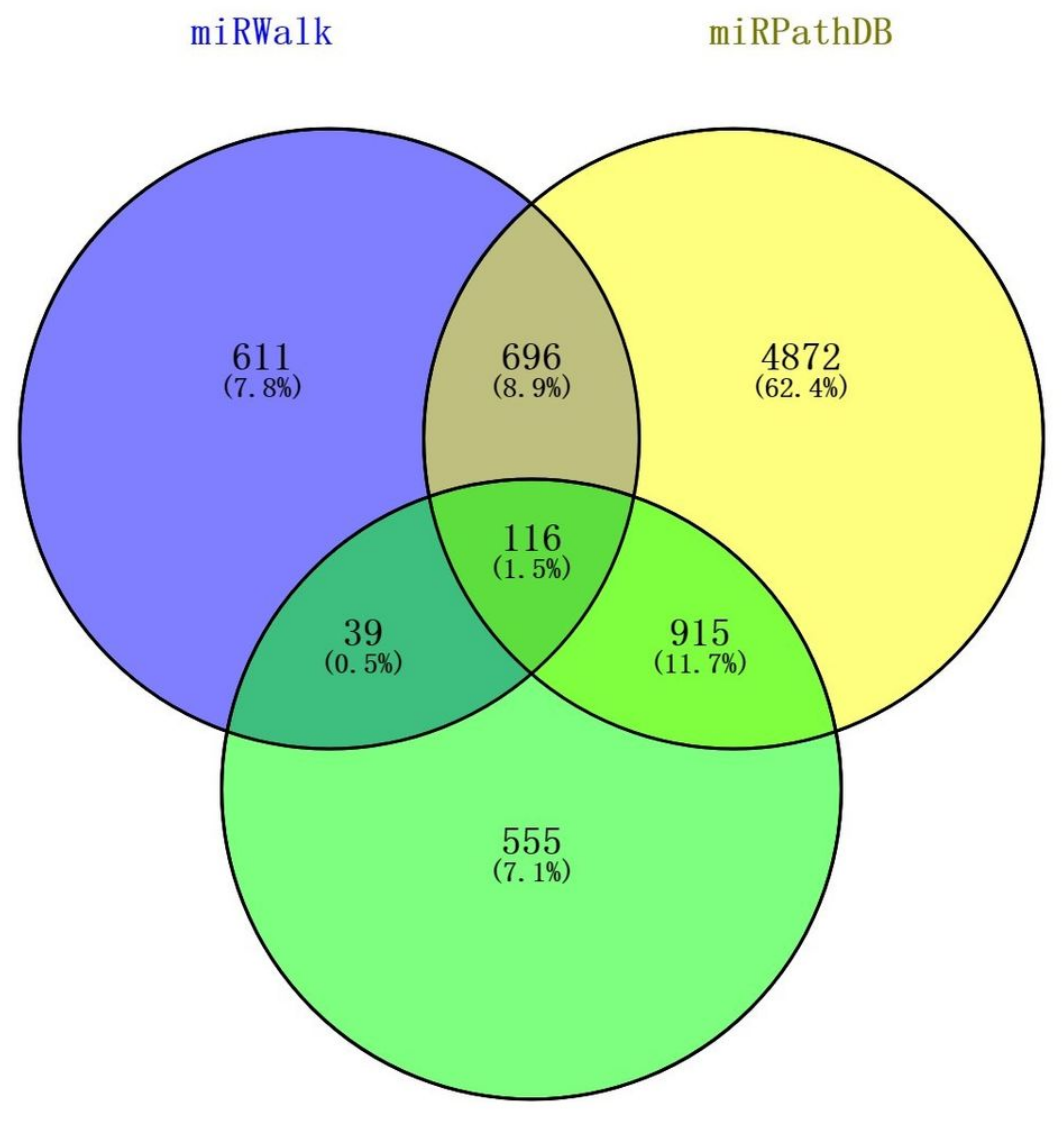

starBase

Figure 6

The intersection results of miRPathDB, miRWalk and starBase 


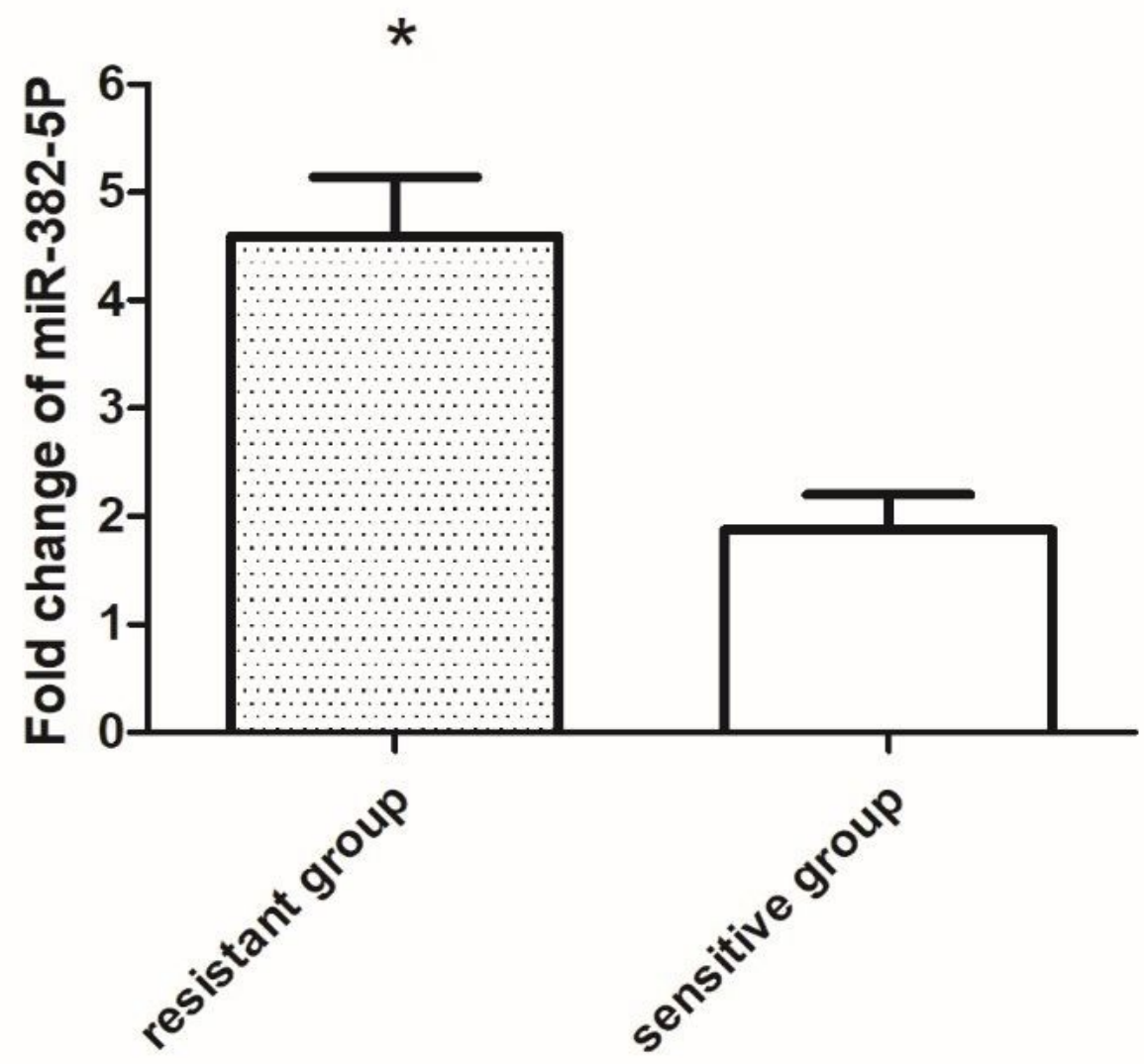

Figure 7

Expression of miR-382-5p in sensitive and drug-resistant ovarian cancer tissues Note ${ }^{*} \mathrm{P}<0.05$

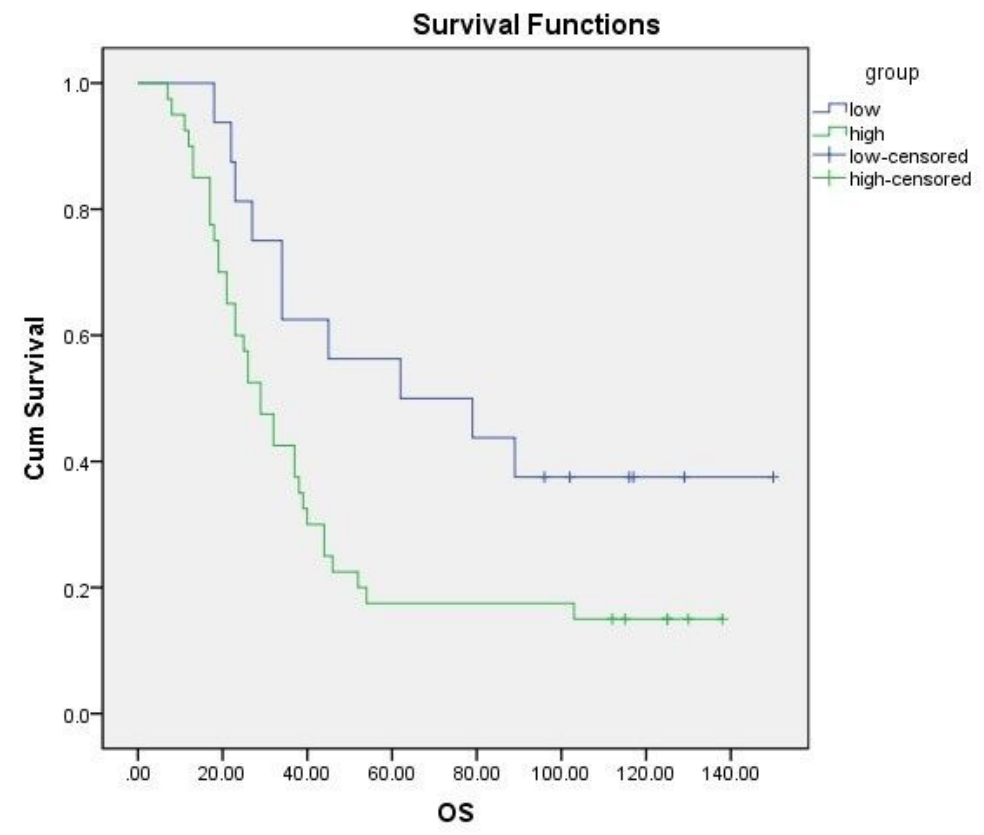

Figure 8

Kaplan-Meier analysis of the correlation between the expression of miR-382-5P and OS in ovarian cancer 


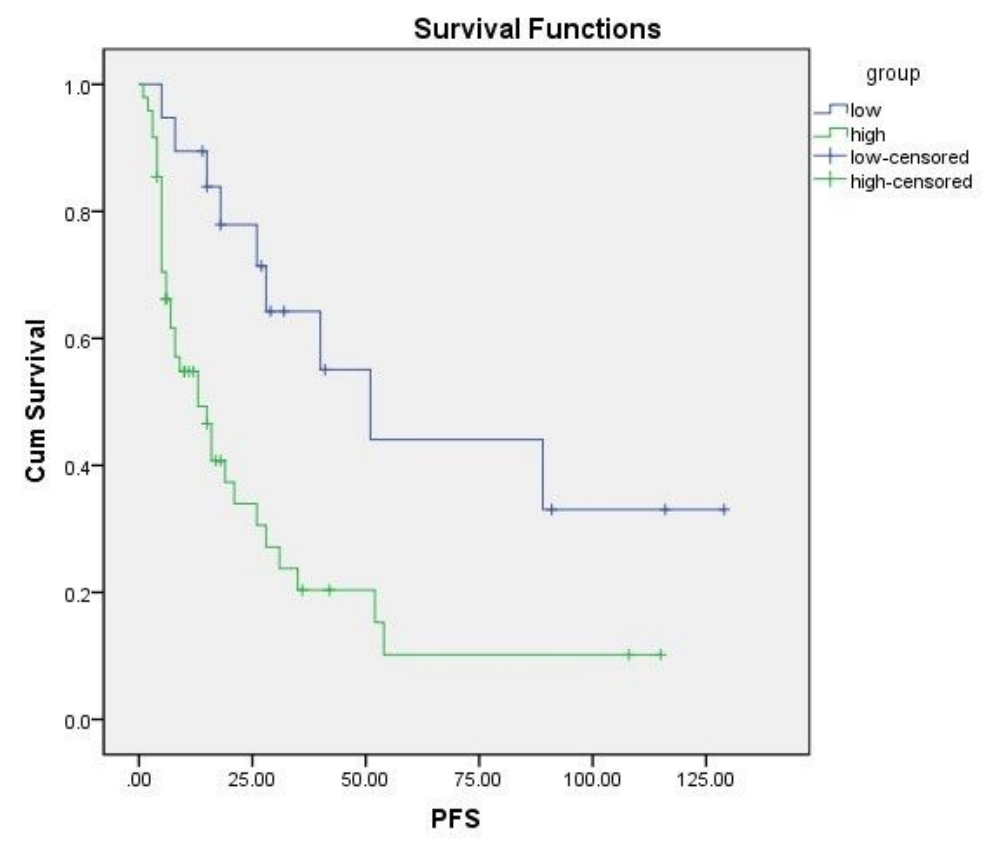

Figure 9

Kaplan-Meier analysis of the correlation between the expression of miR-382-5P and PFS in ovarian cancer

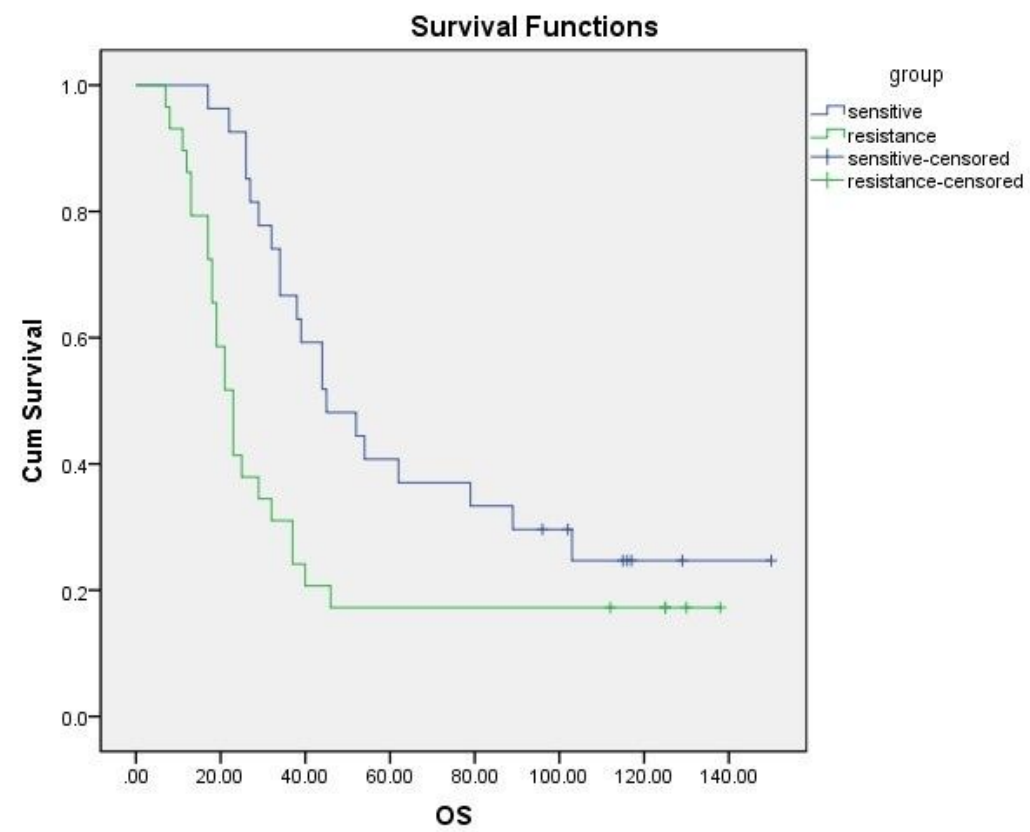

Figure 10

Kaplan-Meier analysis of the correlation between drug resistance and OS in ovarian cancer 


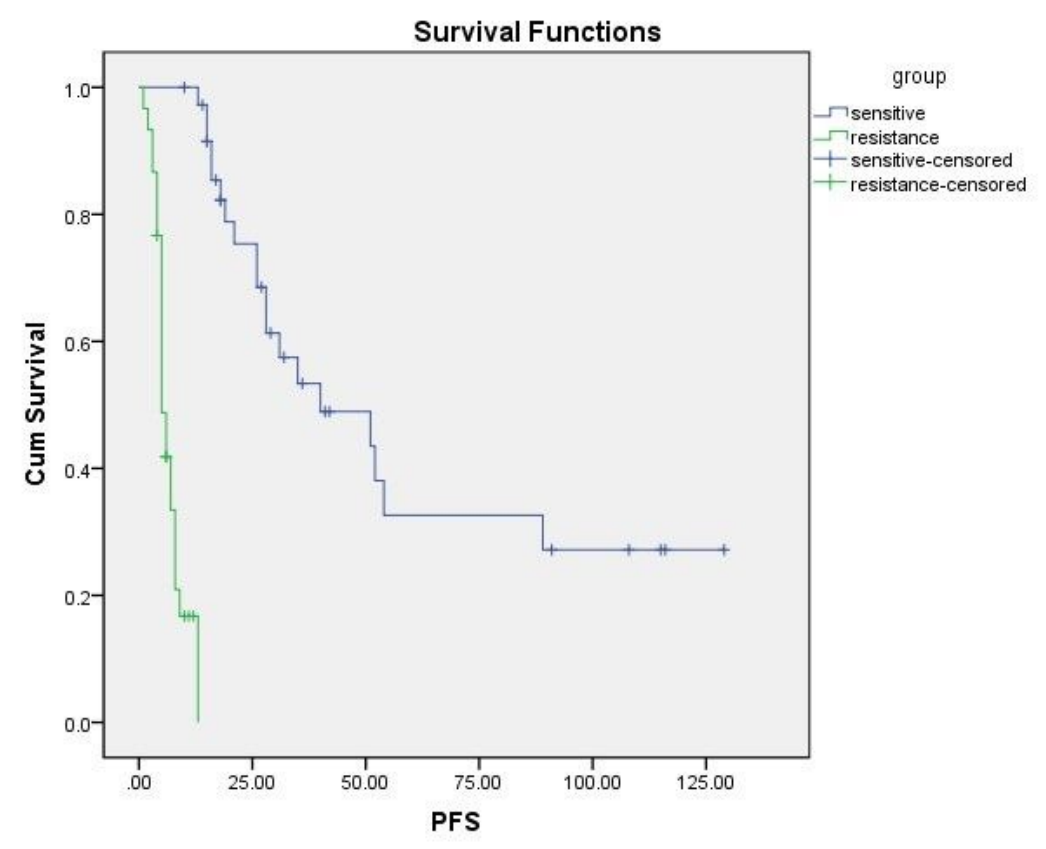

Figure 11

Kaplan-Meier analysis of the correlation between drug resistance and PFS in ovarian cancer

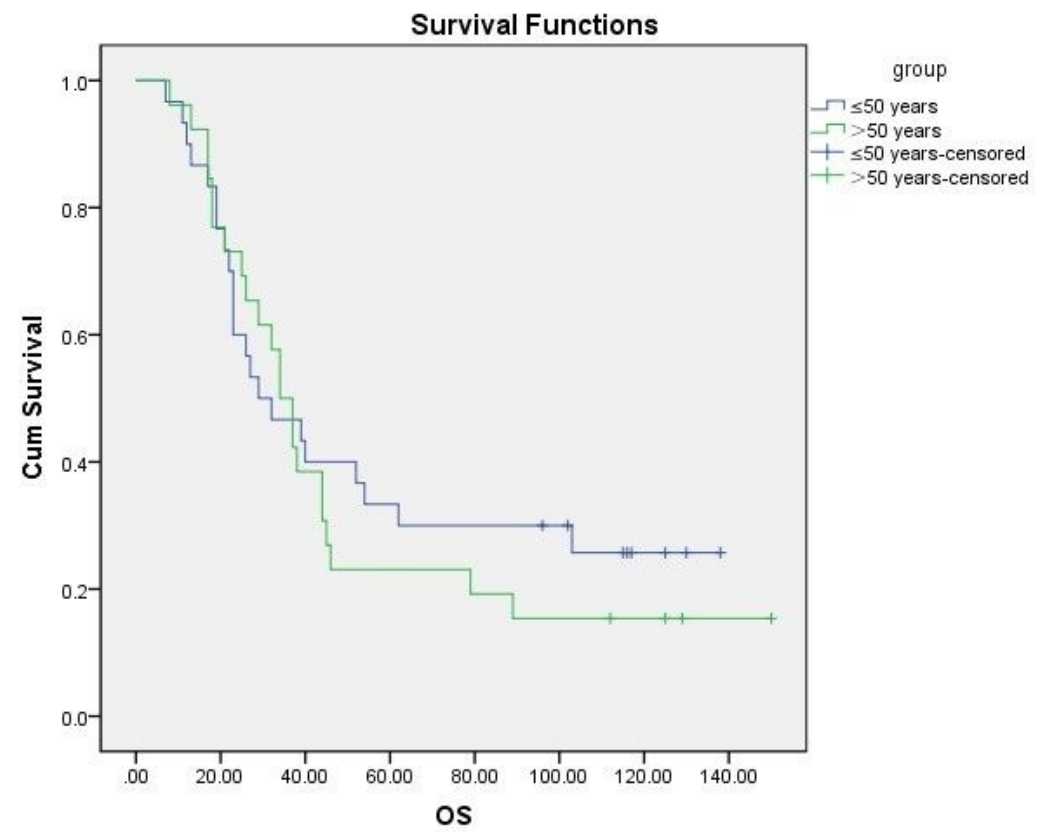

Figure 12

Kaplan-Meier analysis of the correlation between the age of ovarian cancer and its OS 


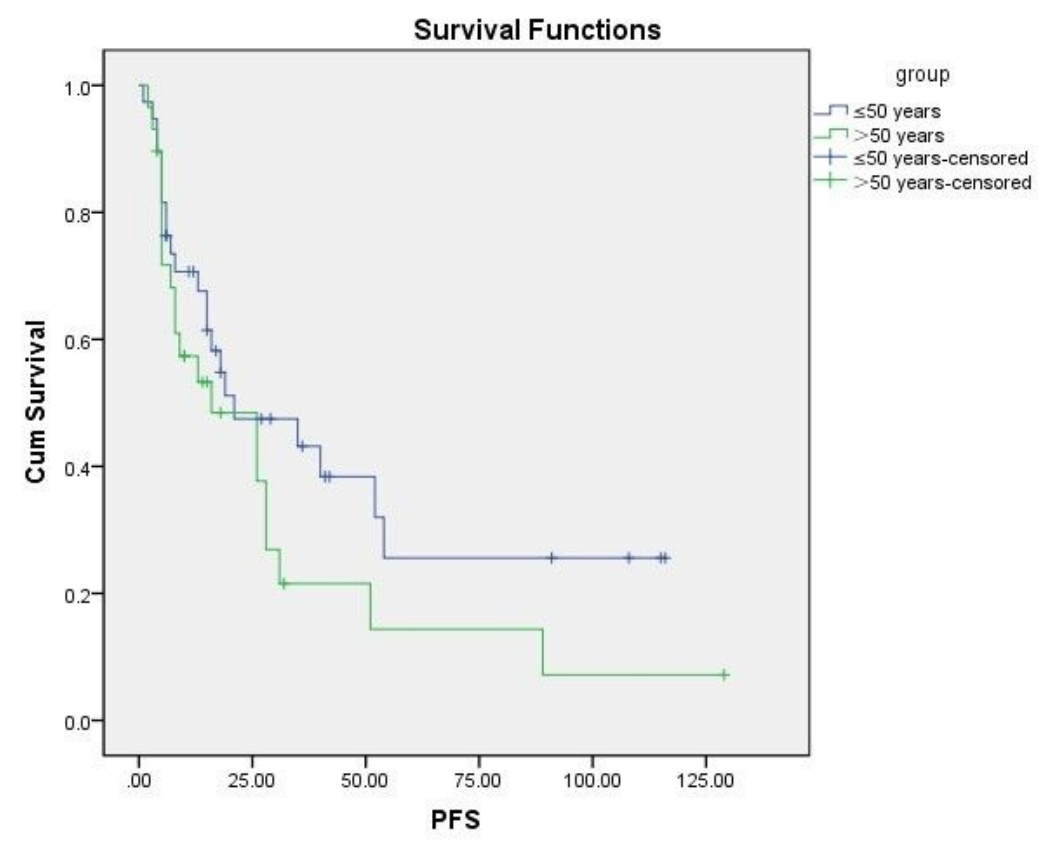

\section{Figure 13}

Kaplan-Meier analysis of the correlation between the age of ovarian cancer and its PFS

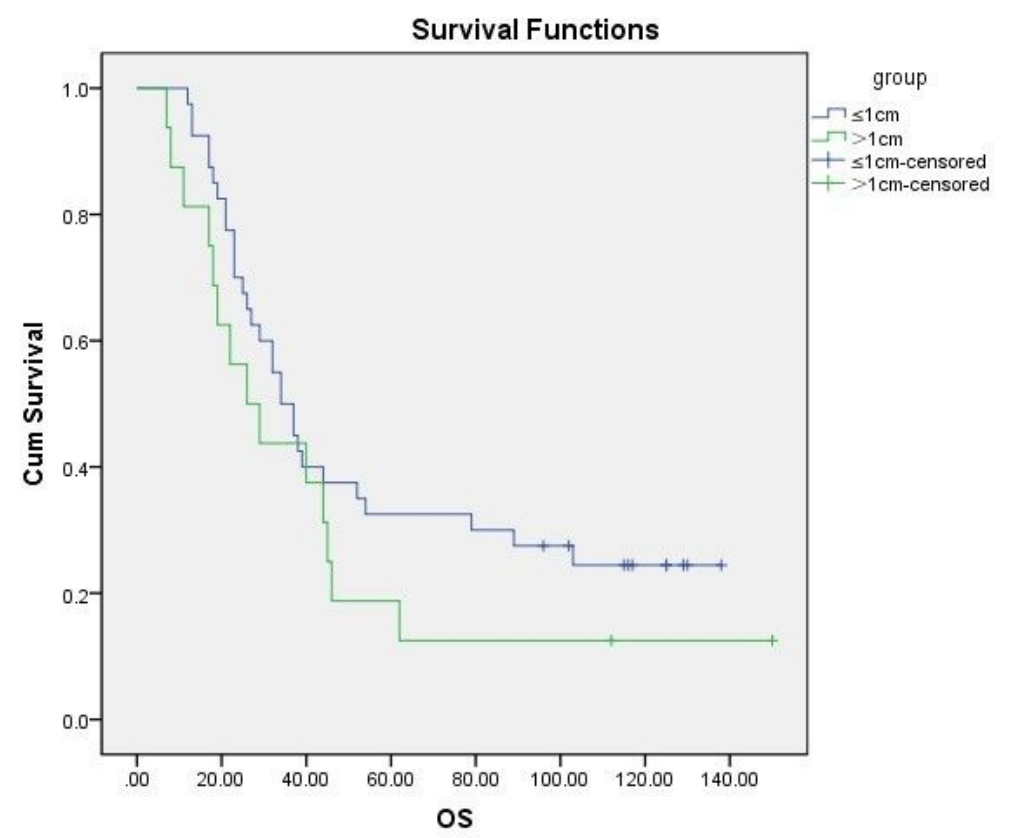

Figure 14

Kaplan-Meier analysis of the correlation between ovarian cancer diameter and OS 


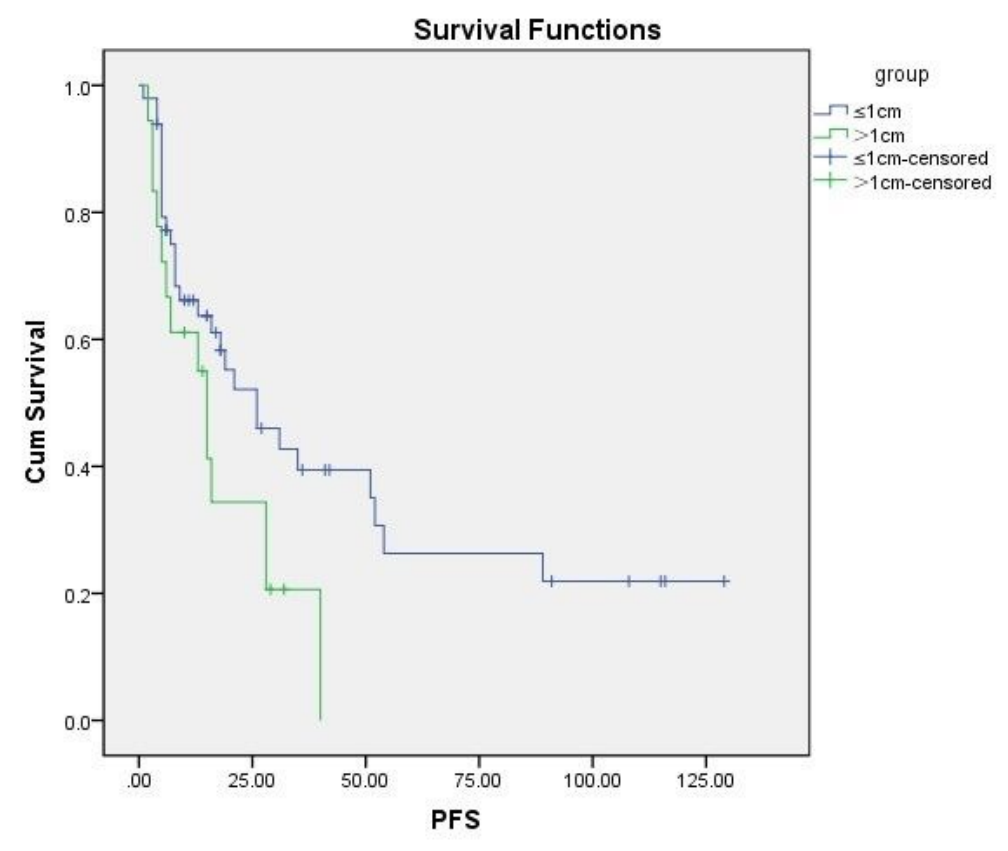

Figure 15

Kaplan-Meier analysis of the correlation between ovarian cancer diameter and PFS

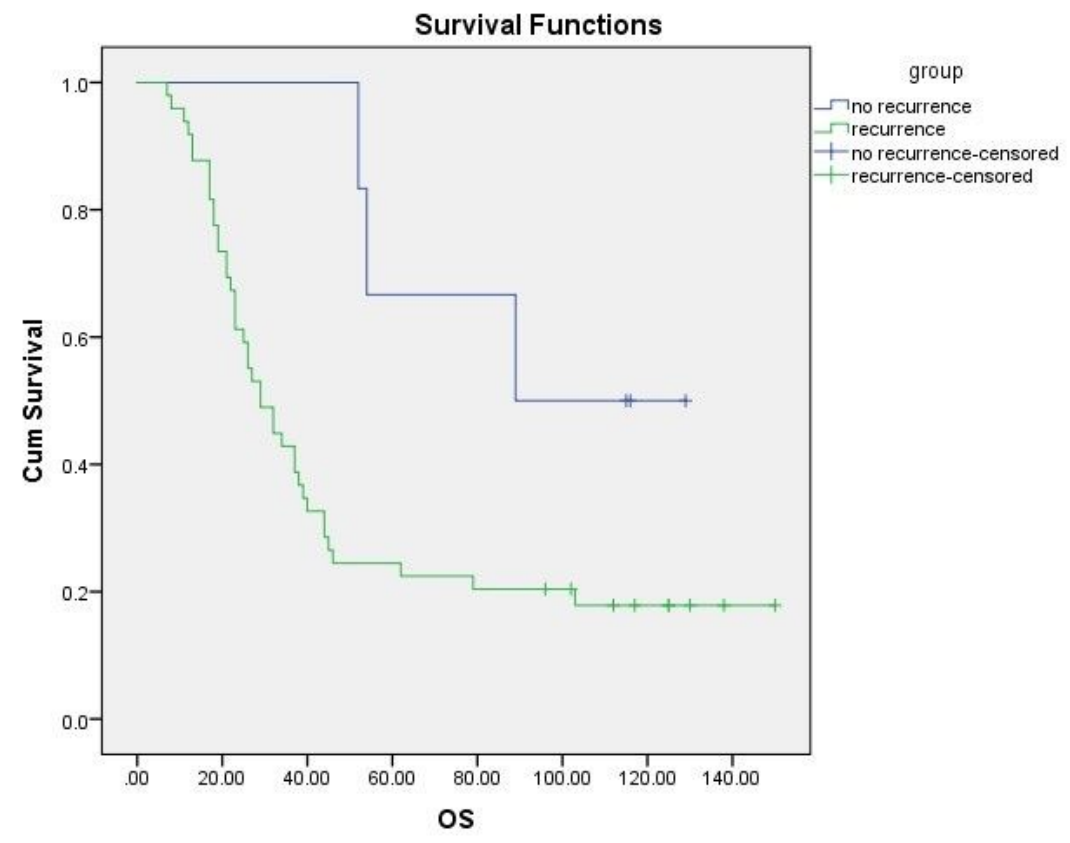

Figure 16

Kaplan-Meier analysis of the correlation between recurrence of ovarian cancer and OS 
Survival Functions

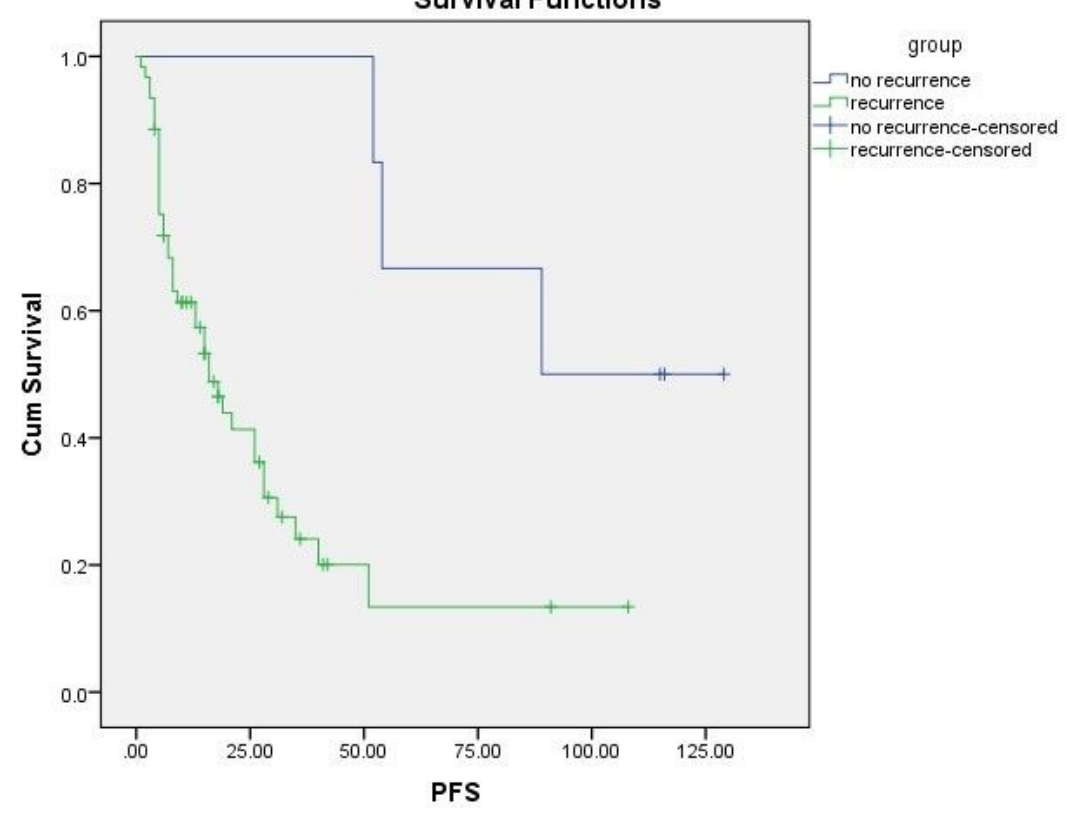

\section{Figure 17}

Kaplan-Meier analysis of the correlation between recurrence of ovarian cancer and PFS

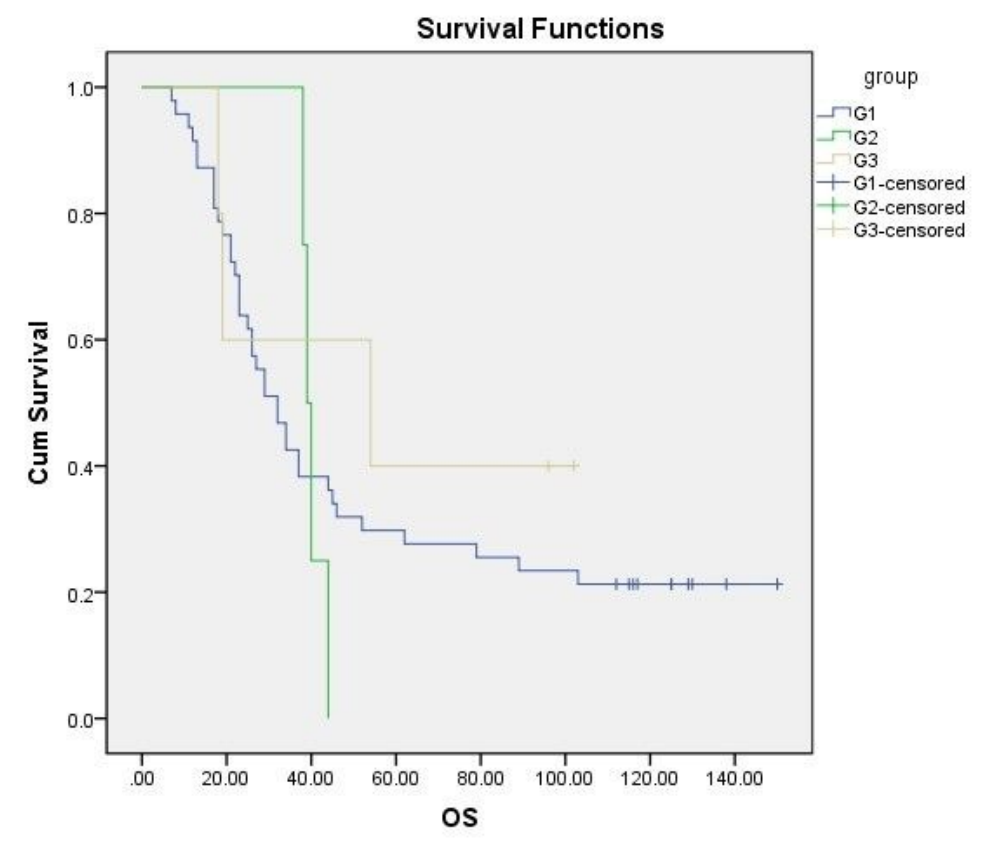

Figure 18

Kaplan-Meier analysis of the correlation between histological grading and OS in ovarian cancer 


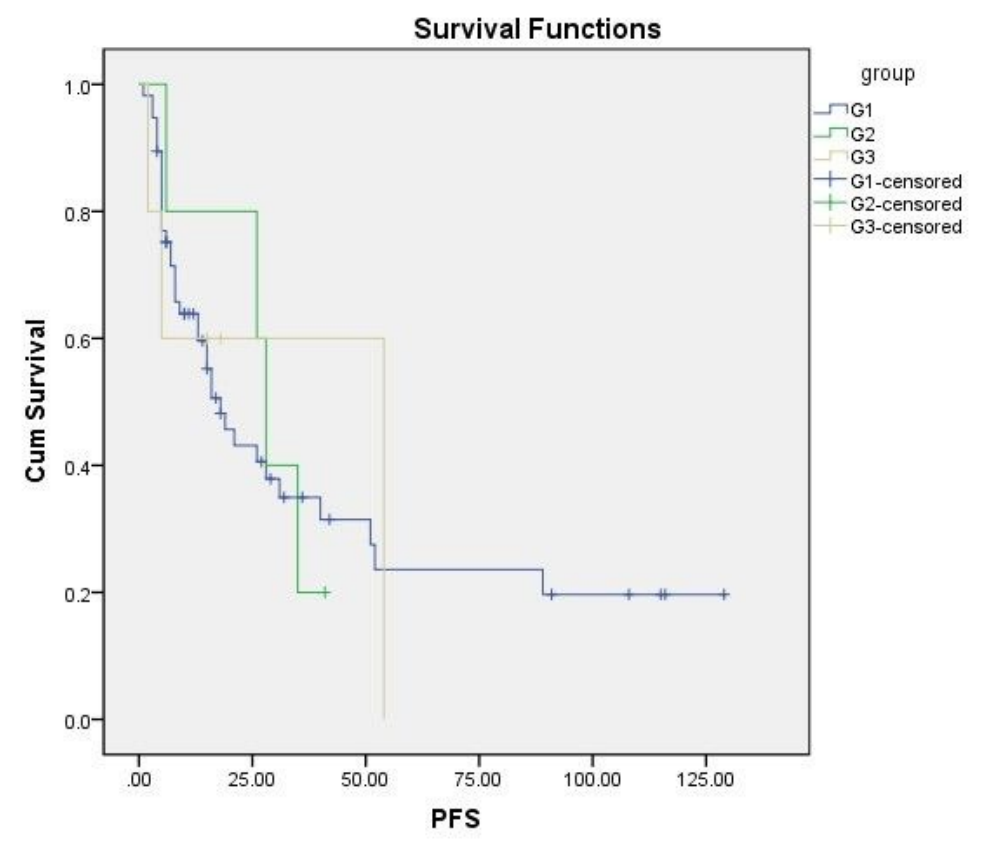

\section{Figure 19}

Kaplan-Meier analysis of correlation between histological grading and PFS in ovarian cancer

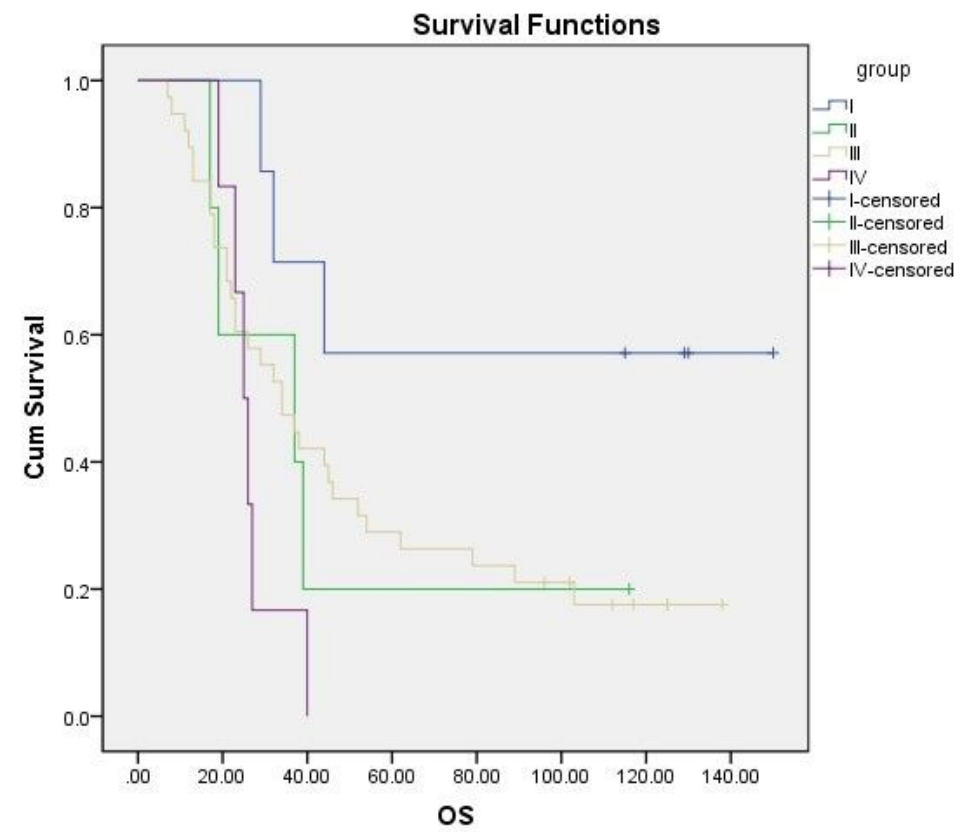

Figure 20

Kaplan-Meier analysis of the correlation between FIGO staging and OS in ovarian cancer 


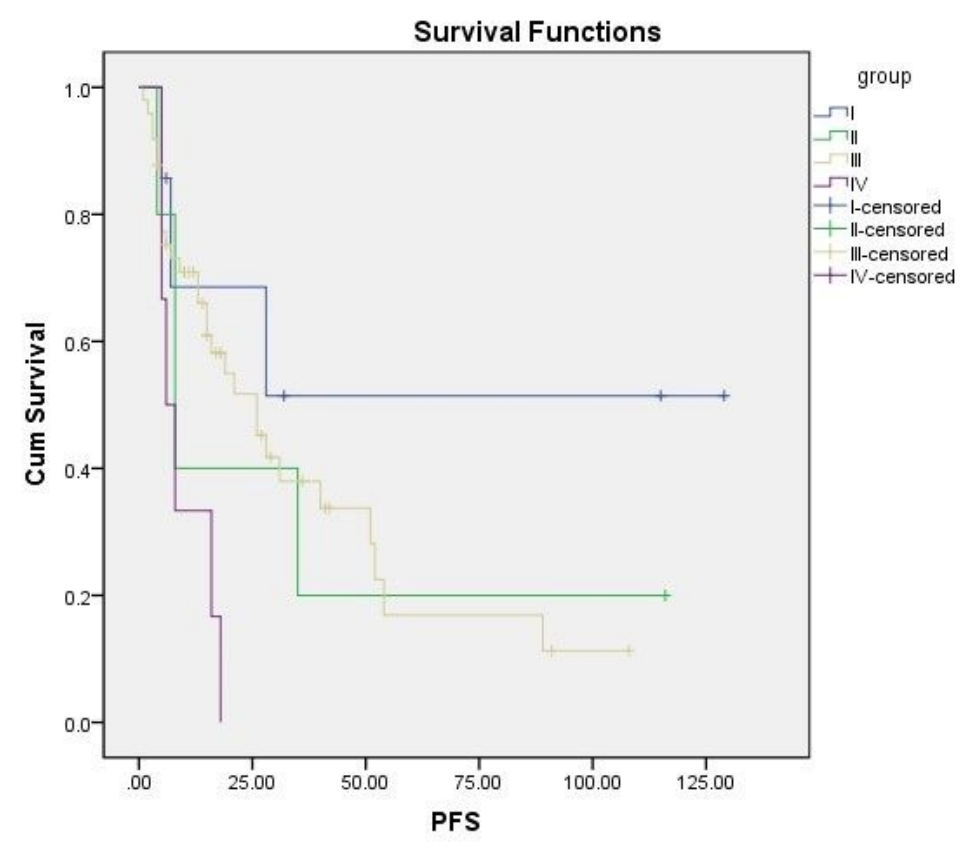

Figure 21

Kaplan-Meier analysis of correlation between FIGO staging and PFS in ovarian cancer

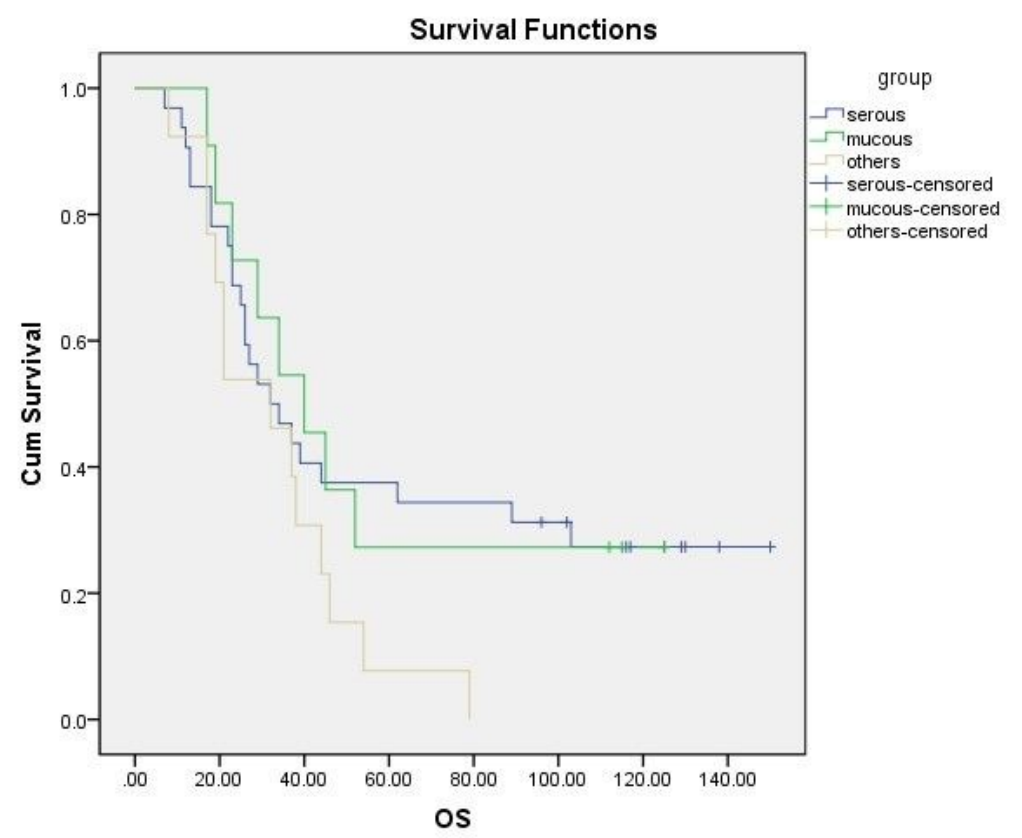

Figure 22

Kaplan-Meier analysis of the correlation between pathological types of ovarian cancer and OS 


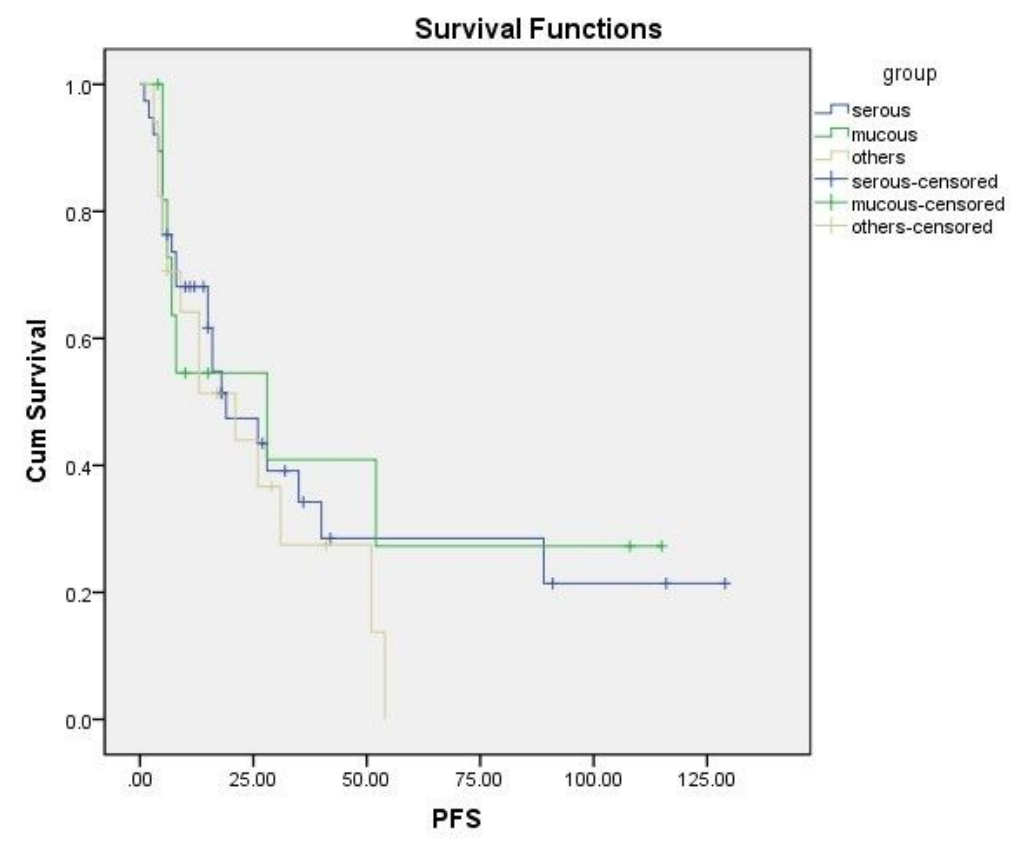

Figure 23

Kaplan-Meier analysis of the correlation between pathological types of ovarian cancer and PFS

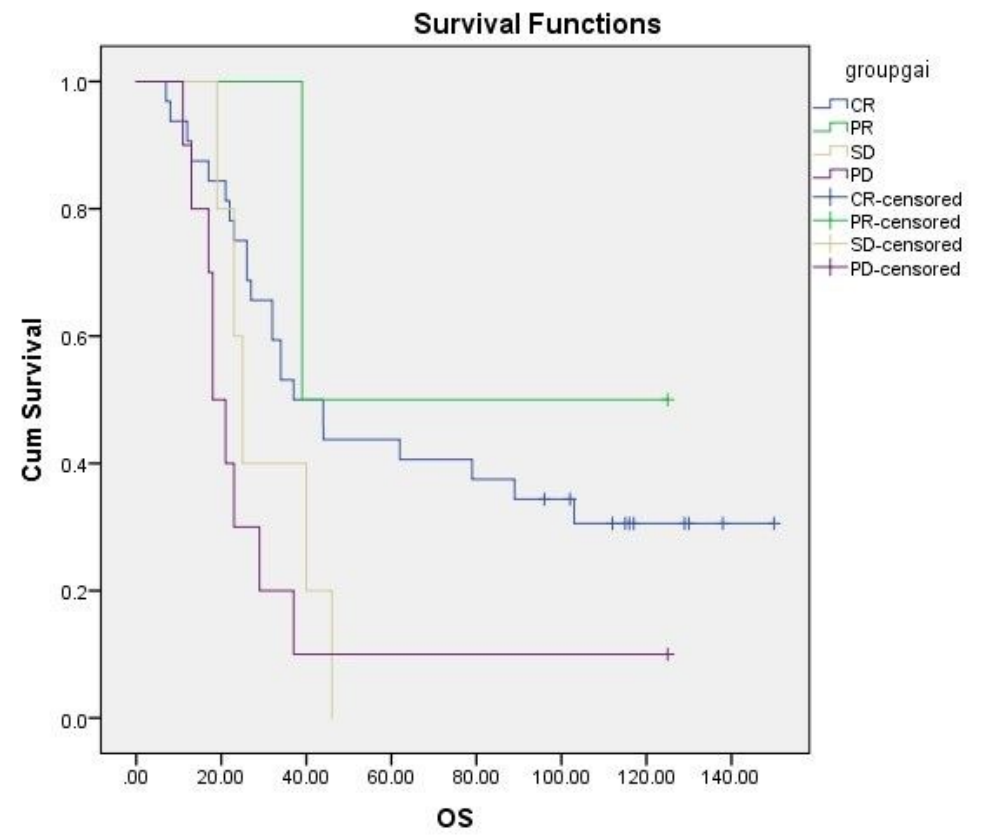

Figure 24

Kaplan-Meier analysis of the correlation between treatment response and OS in ovarian cancer 


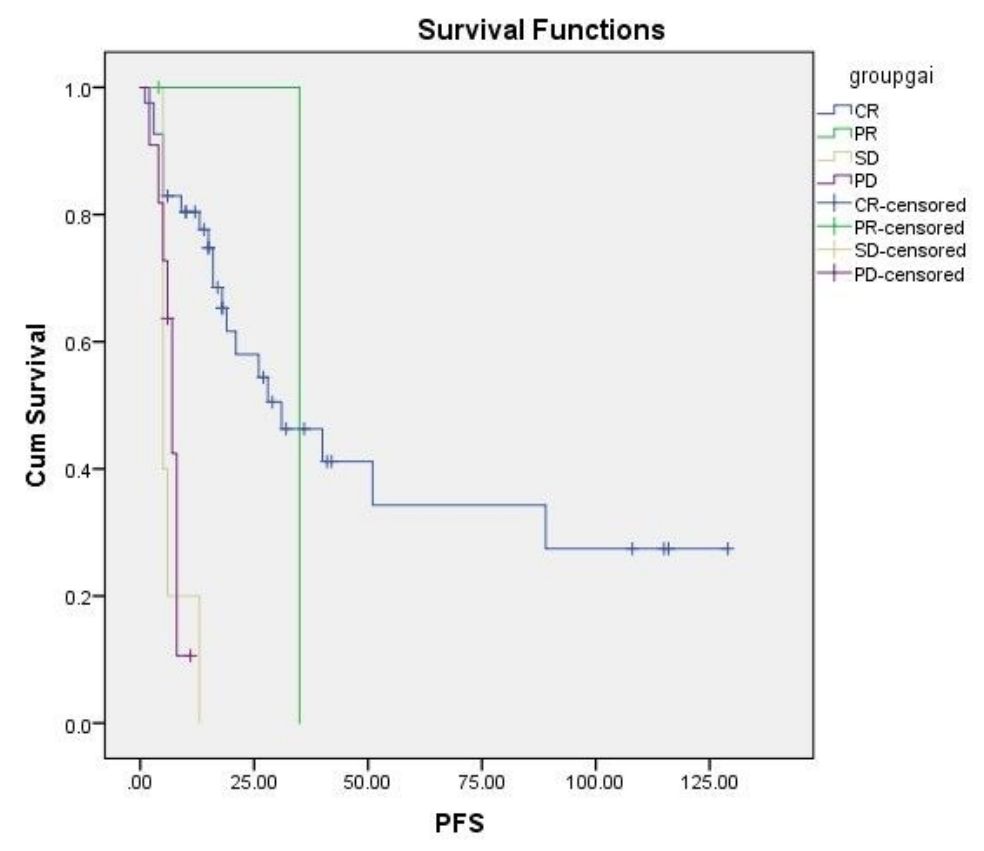

\section{Figure 25}

Kaplan-Meier analysis of the correlation between treatment response and PFS in ovarian cancer

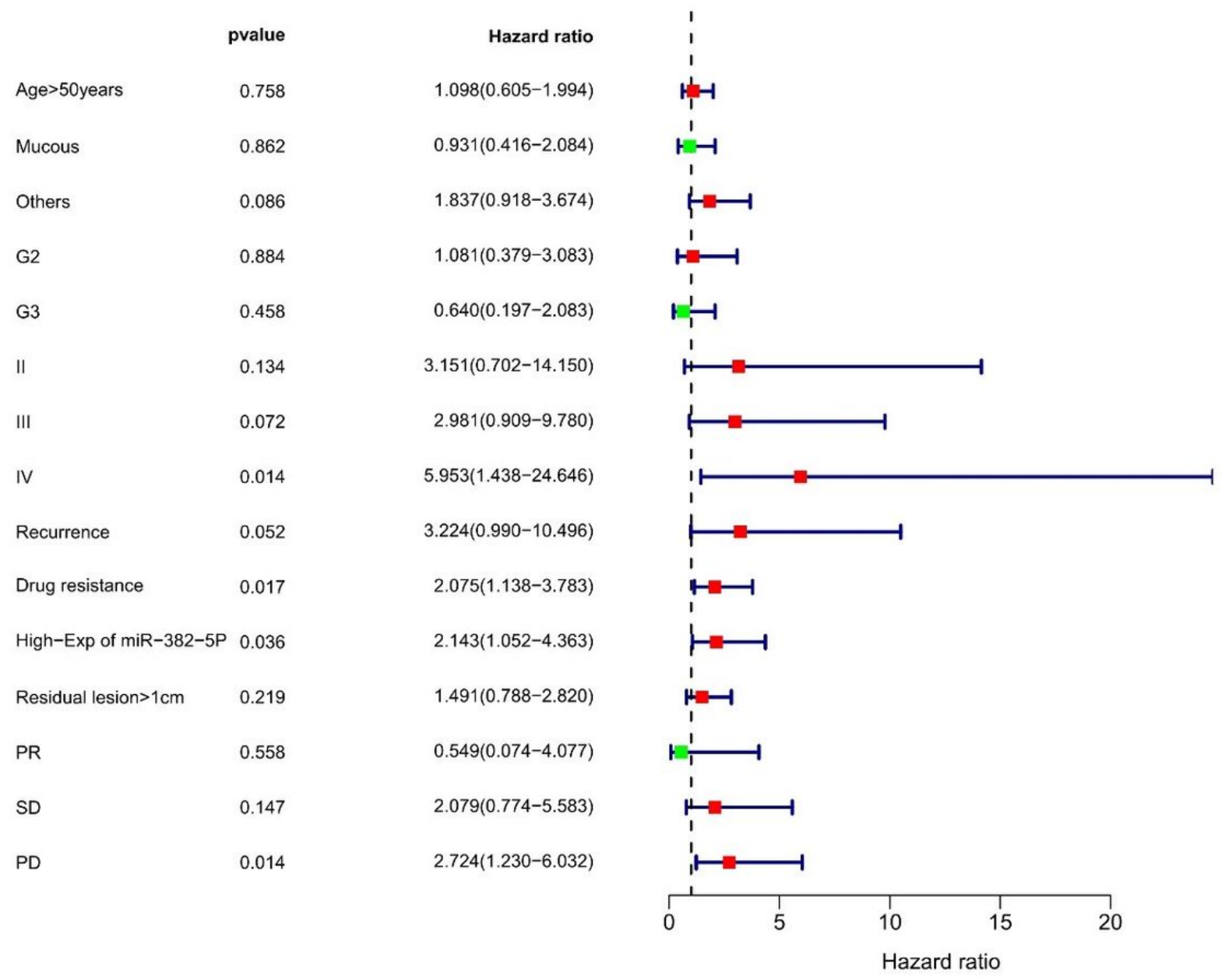

Figure 26 
Cox univariate analysis of prognostic factors of ovarian cancer

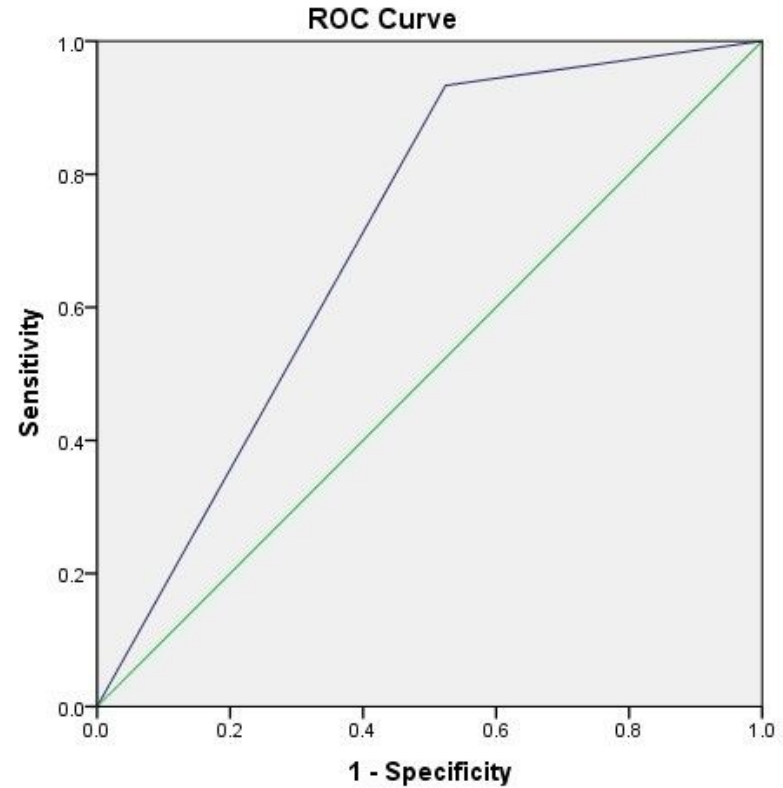

\section{Figure 27}

Diagnostic tests to determine the accuracy of miR-382-5P as an index of drug resistance in ovarian cancer 